ARTICLE

\title{
A protein-independent fluorescent RNA aptamer reporter system for plant genetic engineering
}

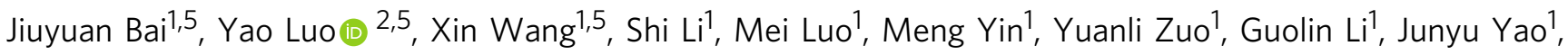

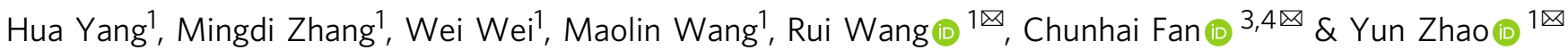

Reporter systems are routinely used in plant genetic engineering and functional genomics research. Most such plant reporter systems cause accumulation of foreign proteins. Here, we demonstrate a protein-independent reporter system, $3 \mathrm{WJ}-4 \times$ Bro, based on a fluorescent RNA aptamer. Via transient expression assays in both Escherichia coli and Nicotiana benthamiana, we show that $3 \mathrm{WJ}-4 \times$ Bro is suitable for transgene identification and as an mRNA reporter for expression pattern analysis. Following stable transformation in Arabidopsis thaliana, 3WJ-4 $\times$ Bro co-segregates and co-expresses with target transcripts and is stably inherited through multiple generations. Further, $3 \mathrm{WJ}-4 \times$ Bro can be used to visualize virusmediated RNA delivery in plants. This study demonstrates a protein-independent reporter system that can be used for transgene identification and in vivo dynamic analysis of mRNA.

\footnotetext{
${ }^{1}$ Key Laboratory of Bio-Resource and Eco-Environment of Ministry of Education, College of Life Sciences, Sichuan University, Chengdu 610065, China. ${ }^{2}$ State Key Laboratory and Collaborative Innovation Center of Biotherapy, West China Hospital, Sichuan University, Chengdu 610041 Sichuan, China. ${ }^{3}$ School of Chemistry and Chemical Engineering, Frontiers Science Center for Transformative Molecules, Institute of Translational Medicine, Shanghai Jiao Tong University, Shanghai 200240, China. ${ }^{4}$ Institute of Molecular Medicine, Shanghai Key Laboratory for Nucleic Acids Chemistry and Nanomedicine, Renji Hospital, School of Medicine, Shanghai Jiao Tong University, Shanghai 200127, China. ${ }^{5}$ These authors contributed equally: Jiuyuan Bai, Yao Luo, Xin Wang.

凶email: wangray1987@scu.edu.cn; fanchunhai@sjtu.edu.cn; zhaoyun@scu.edu.cn
} 
$\mathrm{P}$ lant genetic manipulation techniques are powerful tools in functional genomics research and crop genetic improvement. Using these techniques, target genes can be transformed into recipient plants to achieve various goals, such as gain or loss-of-function ${ }^{1}$. Marker genes, which are critical to efficient transgene plant development, fall into two categories: selectable marker genes and reporter genes ${ }^{2}$. Selectable marker genes, such as antibiotic resistance and herbicide resistance genes, are used to enrich positive transformed cells based on their ability to confer resistance to toxic substances ${ }^{3}$.

Reporter genes do not provide a selective advantage to cells but rather are used to confirm transgenic events because they allow visual detection of transformed cells and tissues. Although numerous reporter genes, including those encoding fluorescent proteins (FPs), $\beta$-glucuronidase (GUS), and luciferase (Luc) ${ }^{4}$, have been employed to identify transgenic lines or visualize target gene expression patterns in plants, they all have limitations ${ }^{4,5}$. For example, the green fluorescent protein gene (GFP) is commonly employed to track subcellular localization of proteins ${ }^{6}$; however, the high autofluorescence of GFP in plant organs often makes it inappropriate for large-scale expression analysis at the wholeplant level ${ }^{7}$. The GUS gene is widely used for tissue-specific expression analysis of target proteins because GUS is readily detectable by histochemical staining ${ }^{8}$, but it cannot be used to measure the dynamic expression of target genes because of the destructive histochemical staining and destaining procedure involved in its detection ${ }^{9}$. The Luc gene has also been frequently used to monitor real-time gene expression in plant ${ }^{10-12}$; however, when measured based on bioluminescence, the Luc activity can be easily affected by substrate availability or inherent differences in local cell environments, therefore making the Luc gene unsatisfactory for accurately monitoring tissue- or cell-specific expression patterns ${ }^{13}$.

Although these reporters can be used for selection of multiple generations of transgenic plants, these systems do not directly detect the presence of the target gene in progeny generations, in which the partial integration of T-DNA may occur ${ }^{14}$; moreover, the expression levels of target genes are not directly reflected by those of the reporter genes. Therefore, PCR and real-time quantitative PCR (qRT-PCR), as well as northern blotting, must be used to reconfirm the status of transgenic lines, which is laborious and time consuming. Conversely, a common approach to characterize the expression pattern of a target gene is to use the gene's native promoter to drive the expression of a reporter gene, but this does not accurately reflect the normal target gene expression pattern because some expression regulatory elements may occur outside of the cloned promoter region ${ }^{15,16}$. In addition, such reporter systems are based on protein products, which can negatively affect gene expression and increase energy utilization in transgenic plants due to the large amount of foreign protein accumulation in cells ${ }^{17}$. Moreover, translational fusions cannot be used to report the expression of non-coding RNA. Consequently, there is a need for a reporter system to directly monitor the expression of different categories of target genes without the accumulation of foreign proteins.

To accomplish this, we focused on reporter systems acting at the transcriptional level. An RNA-based reporter system approach could circumvent some of the limitations of protein-dependent reporter systems. In creating such systems, the greatest challenge is that of exploiting unique markers to allow dynamic imaging of RNAs in living plant cells and tissues. Reif et al. fused the malachite green aptamer, the ribozyme and a siRNA to the bacteriophage phi29 packaging RNA three-way junction (3WJ) motif to generate RNA nanoparticles, which was demonstrated an excellent tool for monitoring RNA folding and degradation in real time in living cells ${ }^{18}$. Besides, several RNA aptamers, with names such as
Spinach and Broccoli, have been also created for live-cell imaging of RNA based on a small fluorophore whose fluorescence is activated upon binding and sequestration within the aptamer ${ }^{19,20}$. Spinach and Broccoli both produce green fluorescence, but Broccoli is superior to Spinach due to the shorter sequence, brighter fluorescence, and higher affinity to fluorophore, (Z)-4-(3,5-difluoro-4-hydroxybenzylidene)-2-methyl-1-(2,2,2-trifluoroethyl)- $1 H$-imidazol $-5(4 H)$-one (DFHBI-1T) ${ }^{21}$. Recently, several aptamers such as Pepper ${ }^{22}$ and Mango $\mathrm{II}^{23}$, based on other fluorophores, have been reported for live-cell imaging of RNA. These reports showed RNA aptamers possess application prospect for RNA imaging in transiently transformed Escherichia coli and mammalian cells ${ }^{24-27}$. Huang et al. designed a Spinach aptamer RNA-based sensor, which is successfully used for quantification and localization of small RNAs in mammalian cells. They also tried to express this RNA aptamer in protoplasts and Nicotiana benthamiana leaves but no reliable signal was detected ${ }^{28}$. So far, there is no report of any aptamer that can be used for RNA imaging in live plant cells. Therefore, we set out to create fluorescent aptamers to apply the RNA aptamer approach to plants, aiming to develop a reporter system for plant genetic engineering at the transcriptional level.

In this study, we design a series of fluorescence aptamers based on the modified $3 \mathrm{WJ}$ scaffold and the optimized Broccoli sequence (Bro). We investigate the performance of the $3 \mathrm{WJ}-\mathrm{nBro}$ series and the previously developed F30-Bro in RNA imaging both in vitro and in vivo. $3 \mathrm{WJ}-4 \times$ Broccoli $(3 \mathrm{WJ}-4 \times$ Bro) shows bright fluorescence in $N$. benthamiana cells, whereas neither F30Bro nor the other aptamers we created produce a detectable signal, revealing $3 \mathrm{WJ}-4 \times$ Bro to be a satisfactory aptamer for mRNA imaging in plant cells. Next, we use $3 \mathrm{WJ}-4 \times$ Bro as a gene marker in creating a system for reporting the presence and expression of target genes at the transcriptional level in plants through fluorescence visualization. Our results show that $3 \mathrm{WJ}$ $4 \times$ Bro can report the transient expression of target genes in plant cells at the transcriptional level, obviating the need for accumulation of foreign proteins. Furthermore, we establish that $3 \mathrm{WJ}-$ $4 \times$ Bro is a useful marker for the identification of stably transformed Arabidopsis thaliana and for expression analysis of target genes in different tissues but does not perturb the processes of transcription and translation. Notably, $3 \mathrm{WJ}-4 \times$ Bro can be cosegregated and co-expressed with target genes and is then stably inherited through multiple generations of transgenic plants in a Mendelian fashion. In addition, we are able to directly detect the process of RNA delivery in N. benthamiana using our reporter system combined with a modified $N$. benthamiana rattle virus (TRV) system. In summary, we report here the development of a protein-independent reporter system for plant genetic engineering, which should facilitate both functional genomics research and the genetic improvement of crops.

\section{Results}

Construction and screening of RNA fluorescence aptamer. Starting from the previously reported F30-Broccoli ${ }^{2}$ (F30-Bro), we modified the F30 structure to form a $3 \mathrm{WJ}$ and optimized the Broccoli sequence. To screen for an optimum aptamer with strong fluorescence and high stability, we created a series of $3 \mathrm{WJ}-$ nBro aptamers, 3WJ-Broccoli (3WJ-Bro), 3WJ-2 $\times$ Broccoli $(3 \mathrm{WJ}-2 \times$ Bro), $3 \mathrm{WJ}-4 \times$ Bro, and $3 \mathrm{WJ}-6 \times$ Broccoli $(3 \mathrm{WJ}-6 \times$ Bro), based on the modified $3 \mathrm{WJ}$ scaffold and two linkers (Supplementary Fig. 1). We then in vitro transcribed each member of the $3 \mathrm{WJ}$-nBro series, as well as the original F30-Bro, individually with T7 RNA polymerase and incubated them with $10 \mu \mathrm{M}$ DFHBI-1T. The spectra of all the $3 \mathrm{WJ}-\mathrm{nBro}$ series had the same single emission peak at $527 \mathrm{~nm}$ and the same single excitation 

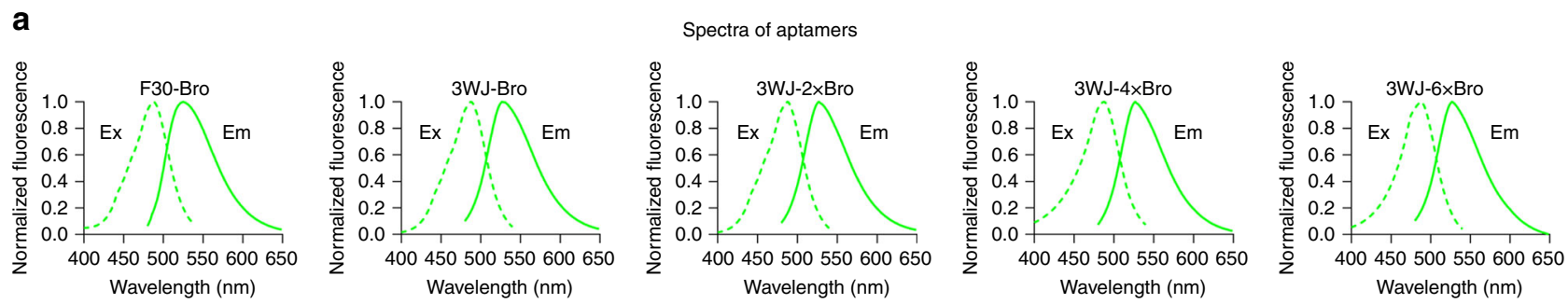

\section{b}
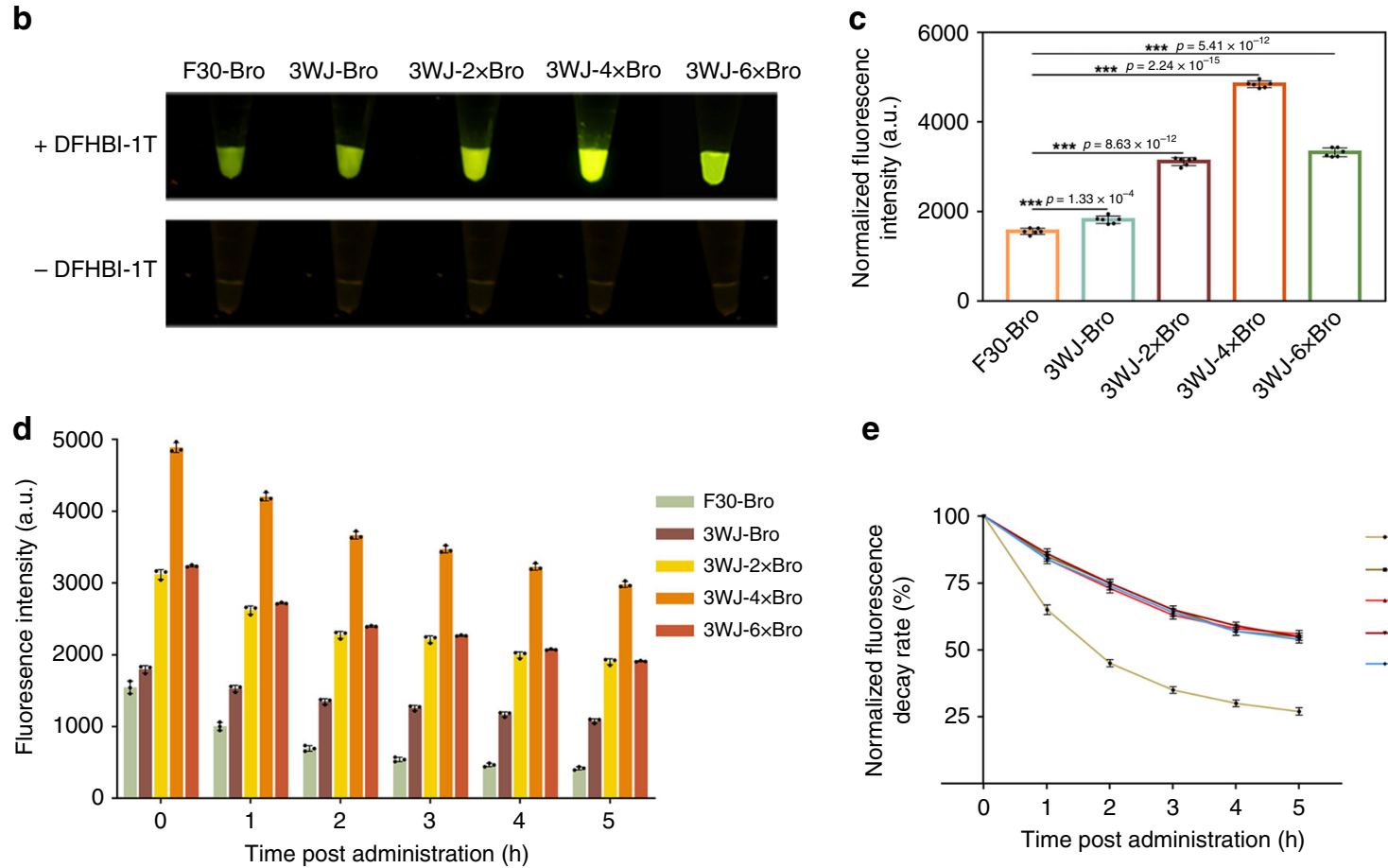

e

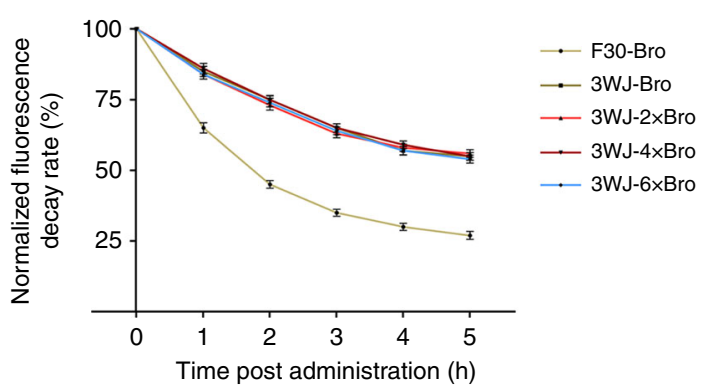

Fig. 1 In vitro performance of the $\mathbf{3 W J}$-nBro series. a Excitation (dashed) and emission (solid) spectra of RNA aptamers. $\mathbf{b}$ Representative fluorescence images of 3WJ-nBro series from a Blue Light Gel Imager (three replicated trials). c Fluorescence quantification of 3WJ-nBro series. Error bars depict the mean \pm standard deviation (s.d.) $(n=6)$ and $p$ values are calculated using an unpaired, two-tailed, Student's $t$ test. ${ }^{\star \star \star} p<0.001$. $\mathbf{d}$ Fluorescence detection within $5 \mathrm{~h}$ after DFHBI-1T administration $(n=3)$. e Normalized fluorescence decay rate calculated as the ratio of remaining fluorescence to initial fluorescence $(n=3)$. All experiments above were repeated three independent times. Source Data are provided as a Source Data file.

\begin{tabular}{|c|c|c|c|}
\hline Aptamers & $\begin{array}{l}\text { Optimal } \\
\text { excitation (nm) }\end{array}$ & $\begin{array}{l}\text { Optimal } \\
\text { emission (nm) }\end{array}$ & $\begin{array}{l}\text { Extinction } \\
\text { coefficient } \\
\left(\mathrm{M}^{-1} \mathrm{~cm}^{-1}\right)\end{array}$ \\
\hline F30-Bro & 485 & 525 & 14,580 \\
\hline 3WJ-Bro & 488 & 527 & 19,490 \\
\hline $3 \mathrm{WJ}-2 \times \mathrm{Bro}$ & 488 & 527 & 31,870 \\
\hline $3 \mathrm{WJ}-4 \times$ Bro & 488 & 527 & 48,390 \\
\hline $3 \mathrm{WJ}-6 \times$ Bro & 489 & 527 & 33,420 \\
\hline
\end{tabular}

peak at $488 \mathrm{~nm}$, similar to the spectrum of the F30-Bro (Fig. 1a and Table 1). The extinction coefficient of all the $3 \mathrm{WJ}$-nBro series was increased compared with F30-Bro, among which the extinction coefficient of $3 \mathrm{WJ}-4 \times$ Bro was the highest, reaching $48390 \mathrm{M}^{-1} \mathrm{~cm}^{-1}$ (2.32-fold higher than that of F30-Bro) (Table 1). All of the aptamer RNAs produced green fluorescence signals after excitation with $488-\mathrm{nm}$ light, and the $3 \mathrm{WJ}-\mathrm{nBro}$ series exhibited higher brightness than F30-Bro (Fig. 1b). Quantitative fluorescence data showed significantly higher fluorescence intensity for 3WJ-Bro compared with F30-Bro, indicating that our $3 \mathrm{WJ}$ scaffold improved the fluorescence intensity of Broccoli (Fig. 1c). Furthermore, the fluorescence intensities of $3 \mathrm{WJ}-2 \times$ Bro and $3 \mathrm{WJ}-4 \times$ Bro were 0.5 -fold and 1.2-fold higher, respectively, than that of $3 \mathrm{WJ}$-Bro, whereas that of $3 \mathrm{WJ}-6 \times$ Bro was lower than that of $3 \mathrm{WJ}-4 \times$ Bro but 0.7 -fold higher than that of $3 \mathrm{WJ}$-Bro. Thus, embedding multiple tandemrepeated Broccoli units in the $3 \mathrm{WJ}$ scaffold and linker did promote fluorescence intensity, and embedding four Broccoli tandem repeats in the scaffold was optimal (Fig. 1b, c). To compare the stability of the four 3WJ-nBro series and F30-Bro, we measured the fluorescence of each aptamer-DFHBI-1T complex at 488-nm excitation every hour for $5 \mathrm{~h}$. The remaining fluorescence was higher for $3 \mathrm{WJ}-\mathrm{nBro}$ series than for F3-Bro throughout the $5 \mathrm{~h}$, and the fluorescence of $3 \mathrm{WJ}-4 \times$ Bro was still 2981.68 a.u. after $5 \mathrm{~h}$, which was sixfold and twofold higher than those of F30-Bro and $3 \mathrm{WJ}$-Bro, respectively (Fig. 1d). We also calculated the fluorescence decay rate after normalization to the corresponding initial fluorescence intensity. F30-Bro showed quicker fluorescence decay than the $3 \mathrm{WJ}-$ nBro series: after $5 \mathrm{~h}, 65 \%$ of the initial fluorescence remained for $3 \mathrm{WJ}-4 \times$ Bro, but only $27 \%$ for F 30 -Bro (Fig. 1e). However, no significant difference of fluorescence decay rate was detected among the $3 \mathrm{WJ}$-nBro series (Fig. 1e). Thus, all 
a

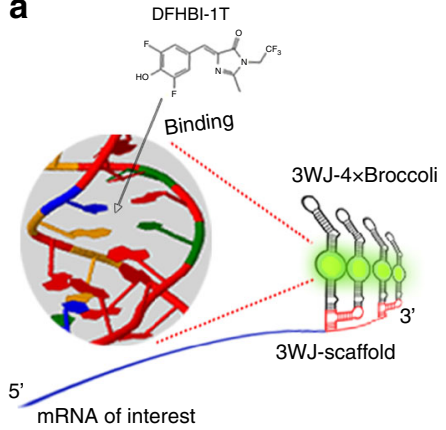

b

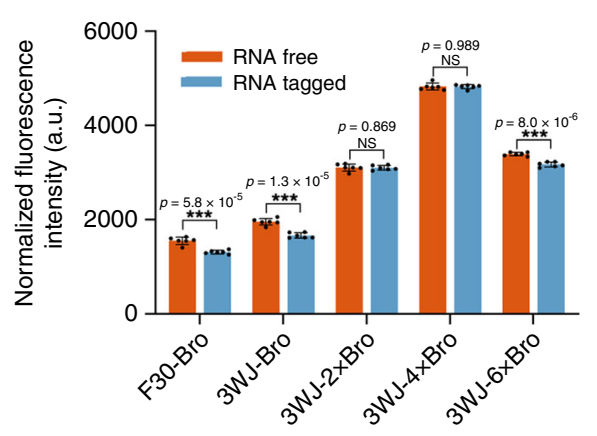

C

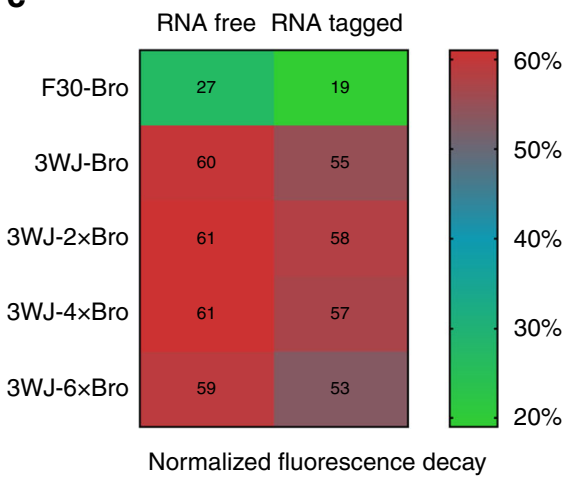

d

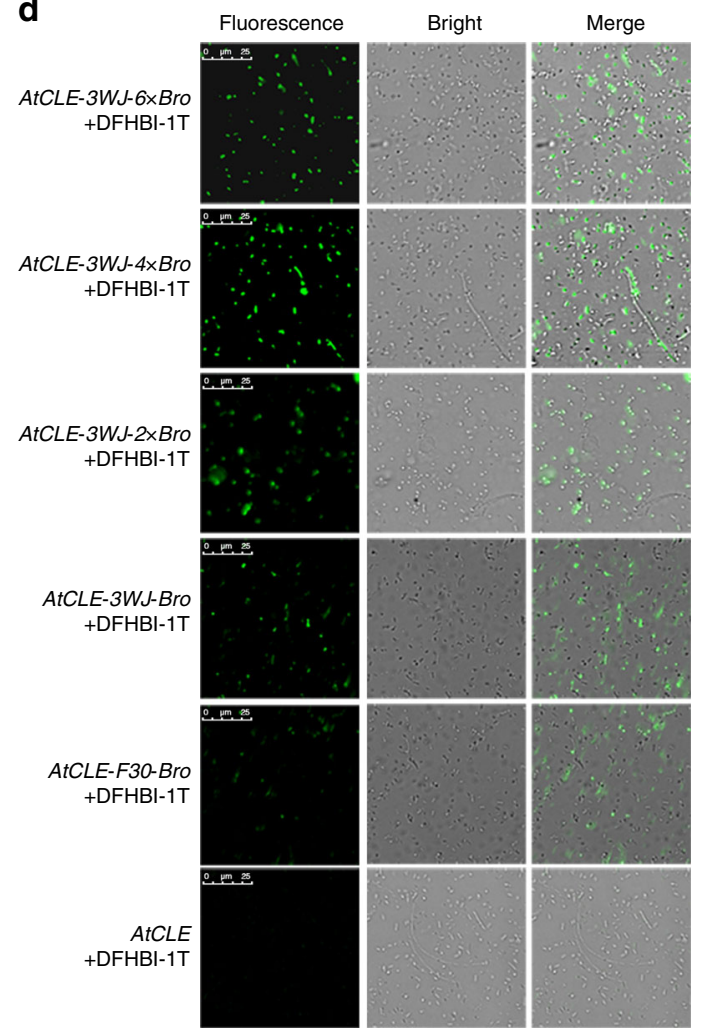

$\mathbf{e}$

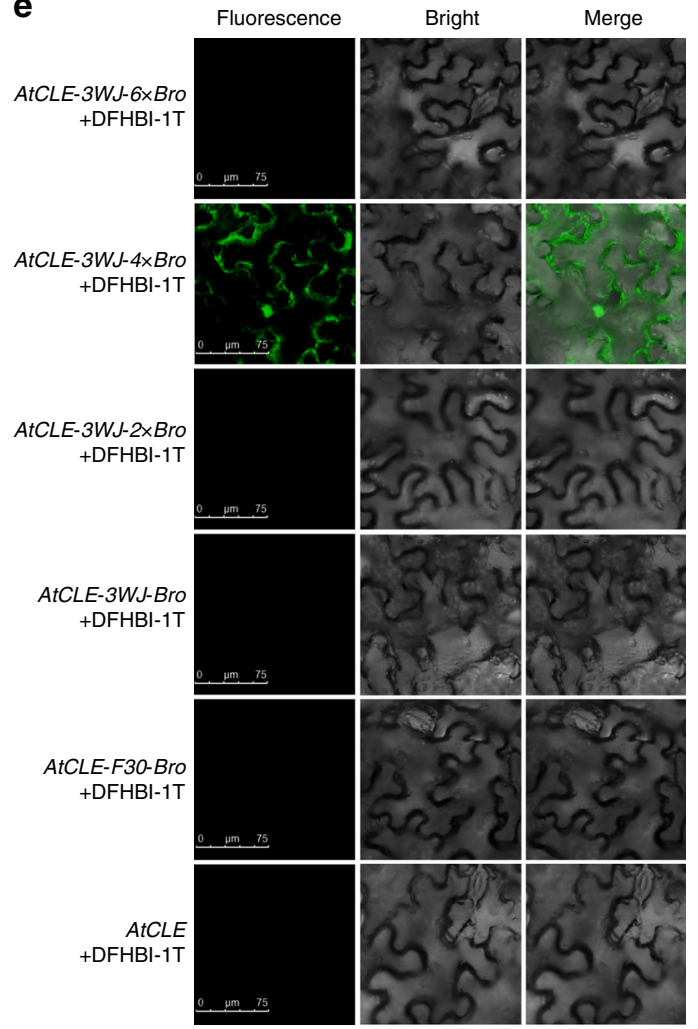

Fig. 2 Comparison of 3WJ-nBro series for imaging mRNA in vitro and in vivo. a Schematic view of mRNA imaging by 3 WJ-nBro series. $\mathbf{b}$ Fluorescence changes of 3WJ-nBro series after appending to the $3^{\prime}$ end of AtCLE mRNA. Error bars depict the mean $\pm \mathrm{s}$.d. $(n=6)$ and $p$ values are calculated using an unpaired, two-tailed, Student's $t$ test. ${ }^{\star \star *} p<0.001$. NS, for not significant. c Differential fluorescence decay of 3 WJ-nBro series with and without AtCLE mRNA $(n=6)$. d Representative fluorescence images of 500 E. coli BL21 cells expressing 3WJ-nBro-tagged AtCLE mRNAs after incubation with $10 \mu M$ DFHBI-1T. Scale bars, $25 \mu \mathrm{m}$. e Fluorescence images of $N$. benthamiana leaf cells expressing $3 \mathrm{WJ}$-nBro-tagged AtCLE mRNAs after incubation with $10 \mu \mathrm{M}$ DFHBI-1T. Scale bars, $75 \mu \mathrm{m}$. All experiments above were repeated three independent times. Source Data underlying b-e are provided as a Source Data file.

four 3WJ-nBro series produce more stable fluorescence than F30Bro, and $3 \mathrm{WJ}-4 \times$ Bro produces the most.

To assess whether the $3 \mathrm{WJ}-\mathrm{nBro}$ series could be used to detect mRNA in vitro, we appended each $3 \mathrm{WJ}$-nBro and F30-Bro downstream of the terminal codon of the target gene AtCLE as a means to transcribe the fusion mRNA in vitro. The target mRNA could be detected from the lighted-up fluorescence of aptamers after DFHBI-1T binding (Fig. 2a). We used the RNAfold web server to predict the minimum free energy (MFE) secondary structures of the five aptamer-tagged mRNAs, which suggested that these aptamers could keep their original MFE structures after being used to tag mRNAs (Supplementary Fig. 2). However, fluorescence imaging and quantification of the fusion mRNAs indicated that F30-Bro, 3WJ-Bro, and $3 \mathrm{WJ}-6 \times$ Bro all had significantly decreased fluorescence intensity when attached to AtCLE mRNA, whereas $3 \mathrm{WJ}-2 \times$ Bro and $3 \mathrm{WJ}-4 \times$ Bro showed no significant effects on fluorescence intensity upon mRNA attachment (Fig. 2b and Supplementary Fig. 3). Comparison of the fluorescence intensities among the fusion mRNAs indicated that AtCLE mRNA tagged with $3 \mathrm{WJ}-4 \times$ Bro $($ AtCLE- $3 W J-4 \times$ Bro) produced the strongest fluorescence signal, of up to 4300 a.u., followed by AtCLE-3WJ-2×Bro, AtCLE-3WJ-6 $\times$ Bro, AtCLE$3 W J-B r o$, and AtCLE-F30-Bro (Fig. 2b). We also assessed the stability of the fluorescence of free and mRNA-fused aptamers, as evidenced by fluorescence decay over $5 \mathrm{~h}$. F30-Bro, 3WJ-Bro, and $3 \mathrm{WJ}-6 \times$ Bro had distinctly lower fluorescence stability when appended to the $3^{\prime}$ terminus of AtCLE mRNA, whereas $3 \mathrm{WJ}-2 \times$ Bro and $3 \mathrm{WJ}-4 \times$ Bro showed similar fluorescence 
stability before and after fusion to the mRNA (Fig. 2c). Consequently, considering both fluorescence intensity and stability, $3 \mathrm{WJ}-4 \times$ Bro showed the greatest potential for use in RNA imaging in vitro among the five aptamers tested.

To explore the performance of $3 \mathrm{WJ}-\mathrm{nBro}$ series in RNA imaging in vivo, we constructed a series of constructs expressing 3WJ-nBro-tagged AtCLE mRNAs and tested them in both E. coli and $N$. benthamiana cells. Confocal microscopy imaging showed that more than $50 \%$ of $E$. coli cells expressing AtCLE-3WJ-2 $\times$ Bro, AtCLE- $3 W J-4 \times$ Bro, or AtCLE- $3 W J-6 \times$ Bro fluoresced, whereas $<30 \%$ of cells expressing AtCLE-3WJ-Bro or AtCLEF30-Bro showed detectable fluorescence (Fig. 2d and Supplementary Fig. 4). In addition, the cells expressing AtCLE-F30-Bro showed considerably weaker average fluorescence than those expressing any of AtCLE-3WJ-nBro mRNAs (Fig. $2 \mathrm{~d}$ and Supplementary Fig. 5), and cells expressing AtCLE-3WJ-4 $\times$ Bro showed the highest fluorescence. Moreover, in N. benthamiana cells, only cells expressing AtCLE-3WJ-4 $\times$ Bro mRNA exhibited bright fluorescence under the confocal microscope, whereas cells expressing any of the other aptamer-tagged mRNAs showed no detectable fluorescence (Fig. 2e). These results indicated that $3 \mathrm{WJ}-4 \times$ Bro was the only fully competent aptamer that could be successfully used for mRNA imaging in both live E. coli cells and plant cells. Consequently, we used $3 \mathrm{WJ}-4 \times$ Bro in constructing our transcriptional-level reporter system.

Characteristics of $3 \mathrm{WJ}-4 \times$ Bro as an in vitro mRNA reporter. The detection of most endogenous RNAs requires highly sensitive imaging probes due to their low expression level. To confirm the detectable level of the $3 \mathrm{WJ}-4 \times$ Bro marker, we designed a series of concentrations for in vitro transcripts of the $3 \mathrm{WJ}-4 \times$ Brotagged AtCLE (AtCLE-3WJ-4 $\times$ Bro), incubated the dilutions with $10 \mu \mathrm{M}$ DFHBI-1T in $50 \mu \mathrm{L}$ HEPES, and imaged them. Fluorescence signals could be observed when the transcript content was as low as $9.375 \mathrm{nmol}$, indicating that $3 \mathrm{WJ}-4 \times$ Bro has high detection sensitivity in mRNA imaging (Supplementary Fig. 6). Furthermore, linear regression showed that the fluorescence intensity increased linearly with increasing transcript contents from 9.375 to $300 \mathrm{nmol}$ (Fig. 3a).

To determine whether $3 \mathrm{WJ}-4 \times$ Bro was a useful marker for reporting the expression of different target genes, we tested three genes of different sequence lengths: AtCLE, mCherry, and NtTuba. We fused $3 \mathrm{WJ}-4 \times$ Bro to the $3^{\prime}$-untranslated region (3'-UTR) of each test gene to create a chimeric gene series (AtCLE-3WJ-4 $\times$ Bro, mCherry-3WJ-4 $\times$ Bro, and NtTuba- $3 W J-$ $4 \times B r o$ ). In vitro imaging of equimolar amounts of transcripts of the three genes showed similar fluorescence levels for each (Fig. 3b), and quantification data showed average fluorescence intensities for each $3 \mathrm{WJ}-4 \times$ Bro-tagged mRNA of more than 4000 a.u., with no significant difference among the three (Fig. 3c), suggesting that $3 \mathrm{WJ}-4 \times$ Bro is sufficiently stable to avoid interference from the different target mRNAs. Results from a fluorescence decay assay showed that $3 \mathrm{WJ}-4 \times$ Bro had greater fluorescence stability after attachment to the $3^{\prime}$-UTR of each of the three mRNAs for $5 \mathrm{~h}$ after adding DFHBI-1T. In addition, we observed an increased fluorescence stability for $3 \mathrm{WJ}-4 \times$ Bro with increasing length of the mRNA sequence over $5 \mathrm{~h}$, indicating that mRNAs with higher molecular weights may enhance the stability of the $3 \mathrm{WJ}-4 \times$ Bro tag (Fig. 3d). These favorable detection sensitivity and stability characteristics make $3 \mathrm{WJ}-4 \times$ Bro a noteworthy potential marker for tracking different mRNAs.

Performance of $3 \mathrm{WJ}-4 \times$ Bro in a prokaryotic expression system. To explore the capability of $3 \mathrm{WJ}-4 \times$ Bro as a marker for reporting functional gene expression in prokaryotic cells, we transformed the constructs harboring the three chimeric genes (AtCLE-3WJ-4 $\times$ Bro, mCherry-3WJ-4 $\times$ Bro, and NtTuba-3WJ$4 \times$ Bro) into E. coli BL21 cells (Supplementary Fig. 7). Confocal imaging showed robust green fluorescence in $E$. coli cells expressing each of the three $3 \mathrm{WJ}-4 \times$ Bro-tagged mRNAs. As a comparison, cells containing either untagged mRNA or DFHBI$1 \mathrm{~T}$ alone showed no detectable fluorescence signal (Fig. 4a and Supplementary Fig. 8). We also assessed the detection efficiency based on the ratio of fluorescent cells to total cells. Approximately $80 \%$ of cells expressing each of the three mRNAs tagged with $3 \mathrm{WJ}-4 \times$ Bro fluoresced, indicative of high detection efficiency for $3 \mathrm{WJ}-4 \times$ Bro in reporting the transcription of different target genes in E. coli cells (Fig. 4b). In addition, quantitation of the fluorescence of cells expressing the three $3 \mathrm{WJ}-4 \times$ Bro-tagged mRNAs showed similar fluorescence intensities in all three, suggesting that the $3 \mathrm{WJ}-4 \times$ Bro marker did not perturb mRNA transcription of any of the three target genes in living $E$. coli cells (Fig. 4c).

Most heterologous RNA aptamers are cleaved by RNase in live cells, causing them to dissociate from the target RNAs. To ensure that the fluorescence observed in $E$. coli precisely reflected the distribution of the mRNA, rather than free $3 \mathrm{WJ}-4 \times$ Bro, we conducted an integrity analysis of the $3 \mathrm{WJ}-4 \times$ Bro-tagged mRNAs by in-gel imaging of fluorescent RNAs, in which total cellular RNAs from induced E. coli cells were separated in a urea denaturing gel and stained with DFHBI-1T. The gel image showed a single band of the expected size for each sample (Fig. 4d). Furthermore, when we excised the RNA bands, eluted them from the gel, and subjected them to reverse transcription sequencing, the cDNA sequence corresponded to the full-length sequence of each $3 \mathrm{WJ}-4 \times$ Bro-tagged mRNA (Supplementary Fig. 9). These results confirmed that the fluorescence signal seen in $E$. coli cells came only from the intact $3 \mathrm{WJ}-4 \times$ Bro-tagged mRNAs, indicating that $3 \mathrm{WJ}-4 \times$ Bro detection accurately reports the expression level of functional genes in prokaryotic cells.

In addition, to explore the effect of $3 \mathrm{WJ}-4 \times$ Bro on the translation activity of target mRNAs, we transformed the different test genes, with and without the $3 \mathrm{WJ}-4 \times$ Bro marker, into E. coli strain Rosetta (DE3) cells for protein expression analysis. The results of sodium dodecyl sulfate-polyacrylamide gel electrophoresis (SDS-PAGE) and Coomassie blue staining showed that cells harboring the test genes both with and without the $3 \mathrm{WJ}-4 \times$ Bro marker expressed target proteins of the expected size and showed the same distribution of total protein on the gel (Supplementary Fig. 10). For further verification, we performed immunoblotting. The target proteins expressed from $3 \mathrm{WJ}-4 \times$ Bro-tagged mRNAs had the same molecular weights and expression levels as those from untagged mRNAs (Fig. 4e). These data confirmed $3 \mathrm{WJ}-4 \times$ Bro as an excellent gene marker for reporting the expression of different target genes in prokaryotic systems that does not perturb either transcription or translation.

Performance of $3 \mathrm{WJ}-4 \times$ Bro in plant transient expression system. The spatiotemporal expression and dynamics of mRNAs play critical roles in directly and indirectly regulating complex biological processes in plants. To further explore the utility of $3 \mathrm{WJ}-4 \times$ Bro as a genetically encoded marker in excised plant cells, we conducted a protoplast transient expression assay. We expressed each of the three $3 \mathrm{WJ}-4 \times$ Bro-tagged mRNAs (AtCLE$3 W J-4 \times$ Bro, mCherry- $3 W J-4 \times B r o$, and NtTub $\alpha-3 W J-4 \times B r o)$ in protoplasts under the control of the CaMV $35 S$ promoter (Supplementary Fig. 11a). Confocal imaging of the protoplasts showed bright fluorescence in those expressing $3 \mathrm{WJ}-4 \times$ Bro-tagged mRNAs after incubation with $10 \mu \mathrm{M}$ DFHBI-1T but no signal in the absence of either $3 \mathrm{WJ}-4 \times$ Bro or DFHBI-1T (Fig. 5a and 
a

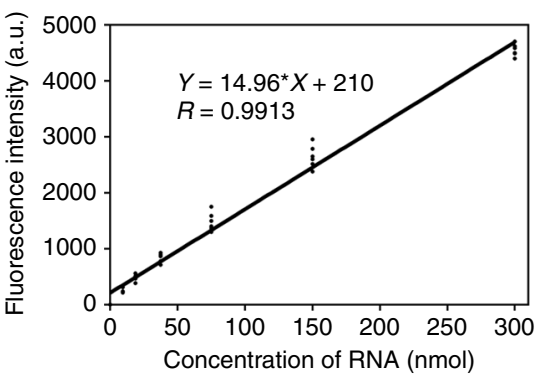

b

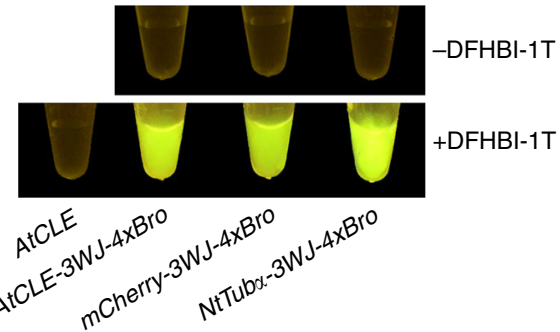

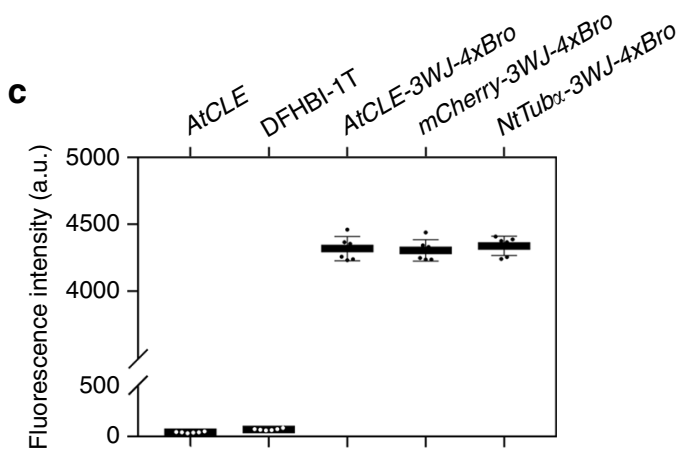

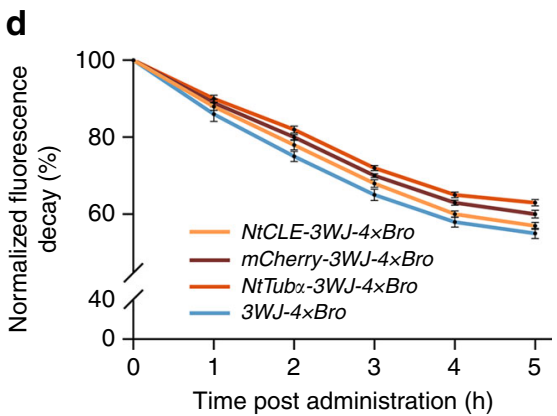

Fig. 3 Characteristics of $\mathbf{3 W J} \mathbf{- 4} \times$ Bro after tagging various mRNAs in vitro. a Linear regression of fluorescence intensity and mRNA content ( $n=6$ ). b Representative image of $300 \mu \mathrm{M} 3 \mathrm{WJ}-4 \times$ Bro-tagged mRNAs in vitro after incubation with $10 \mu \mathrm{M}$ DFHBI-1T. c Quantification of the fluorescence of RNA-DFHBI-1T complexes in $\mathbf{b}$ using a fluorescence spectrophotometer. Error bars, mean \pm s.d. $(n=6)$. $\mathbf{d}$ Comparison of the fluorescence decay of $3 \mathrm{WJ}$ $4 \times$ Bro-tagged mRNAs within $5 \mathrm{~h}$ after DFHBI-1T administration. The fluorescence decay was calculated after normalization of the remaining fluorescence intensity to the respective initial fluorescence intensity. Error bars, mean \pm s.d. $(n=6)$. All experiments were repeated three independent times. Source Data are provided as a Source Data file.

Supplementary Fig. 11b), indicating that $3 \mathrm{WJ}-4 \times$ Bro could be used to report gene expression in plant cells based on the green fluorescence signal. We also observed a distinct cytoplasmic localization of the fluorescence signal for all three $3 \mathrm{WJ}-4 \times$ Brotagged mRNAs (Fig. 5a). Extracellular and intracellular fluorescence detection revealed a high $3 \mathrm{WJ}-4 \times$ Bro fluorescence signal but only low DFHBI-1T background fluorescence (Supplementary Fig. 11c). All three $3 \mathrm{WJ}-4 \times$ Bro-tagged mRNAs showed a high signal-to-background ratio $(\mathrm{S} / \mathrm{B})$ in the green fluorescence channel (Fig. 5b), and quantification showed similar expression levels for all three target genes in protoplasts (Supplementary Fig. 12). Taken together, these results define $3 \mathrm{WJ}-4 \times$ Bro as a favorable marker for reporting mRNA expression in excised plant cells.

To further assess the performance of $3 \mathrm{WJ}-4 \times$ Bro in tracking target gene expression in plant tissues, we introduced the chimeric genes (AtCLE-3WJ-4 $\times$ Bro, mCherry-3WJ-4 $\times$ Bro, and $N t T u b \alpha-3 W J-4 \times B r o$ ) into $N$. benthamiana leaf tissue by Agrobacterium-mediated transformation for transient expression. Confocal imaging showed bright fluorescence in the leaves expressing each of the three target genes with the $3 \mathrm{WJ}-4 \times$ Bro marker after incubation with DFHBI-1T, whereas leaves expressing marker-free genes or not incubated with DFHBI-1T showed no fluorescence (Fig. $5 c$ and Supplementary Fig. 13), confirming that the $3 \mathrm{WJ}-4 \times$ Bro/DFHBI-1T system could be used to report mRNA transcription in plant tissue in vivo. Furthermore, obvious cytoplasmic and nuclear localization signals were observed in leaf cells, demonstrating the distribution of three target mRNAs (Fig. 5c). Scanning of intracellular and extracellular fluorescence intensity (marked by blue lines in Fig. $5 \mathrm{c}$ ) showed high $\mathrm{S} / \mathrm{B}$ in the green fluorescence channel (Fig. 5d), which aided the accuracy of the $3 \mathrm{WJ}-4 \times$ Bro/DFHBI-1T system.

To ensure accurate assessment of the expression levels of target mRNAs, we also detected the integrity of the $3 \mathrm{WJ}-4 \times$ Bro-tagged
mRNAs through an in-gel imaging assay. We observed a single expected band on the gel for each $3 \mathrm{WJ}-4 \times$ Bro-tagged mRNA (Supplementary Fig. 14). Sequencing results of the excised and eluted RNA bands revealed the full-length sequences of each $3 \mathrm{WJ}-4 \times$ Bro-tagged RNA, indicating that the fluorescence came only from the $3 \mathrm{WJ}-4 \times$ Bro-tagged mRNA. Thus, the $3 \mathrm{WJ}-4 \times$ Bro/DFHBI-1T system accurately reported the expression and localization of the target mRNA in plant tissue.

Next, to further investigate the effect of the $3 \mathrm{WJ}-4 \times$ Bro tag on the translation of the fusion mRNAs in plant, we visualized $m$ Cherry- 3 WJ $-4 \times$ Bro mRNA and its protein product in the leaves simultaneously through confocal microscopy. The bright green and red fluorescence signal were simultaneously observed in the $m$ Cherry-3WJ- $4 \times$ Bro channel and the mCherry channel, respectively, indicating that $3 \mathrm{WJ}-4 \times$ Bro did not affect the function of mRNA as a genetically encoded template for protein translation in the plants (Fig. 5e). Moreover, we found no cells showing only green or red fluorescence alone, which indicated that $3 \mathrm{WJ}-4 \times$ Bro was reliably linked to the mRNAs and vice versa. Thus, the spectroscopically and biochemically favorable characteristics of $3 \mathrm{WJ}-4 \times \mathrm{Bro} / \mathrm{DFHBI}-1 \mathrm{~T}$, which include bright, stable fluorescence, high $\mathrm{S} / \mathrm{B}$ contrast, and robust reporting of mRNA expression without perturbation of the target RNA's transcription, localization, or translation, qualify this system as an excellent protein-independent reporter system at the transcriptional level for plant genetic transformation.

To monitor the dynamic process of mRNA synthesis and transfer in plant cells, we transformed the construct expressing AtCLE-3WJ-4 $\times$ Bro into N. benthamiana leaf cells by Agrobacterium infiltration and then incubated leaf samples with $10-\mu \mathrm{M}$ DFHBI-1T at $0,24,48$, and 72 -h post transformation, respectively. The imaging results showed no detectable fluorescence at 0 -h post infiltration. AtCLE- 3 WJ- $4 \times$ Bro fluorescence 
a
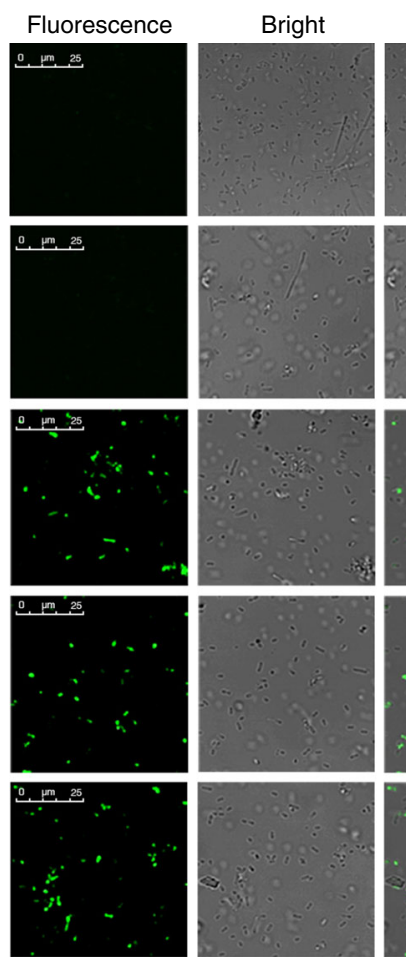

d

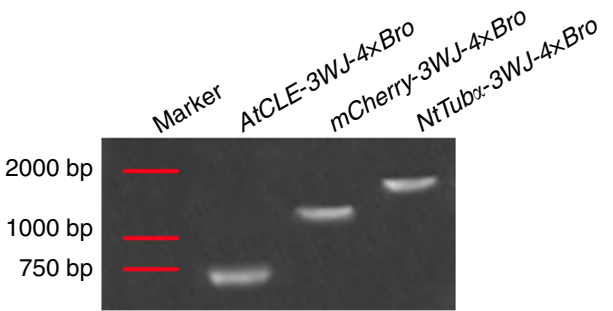

Merge
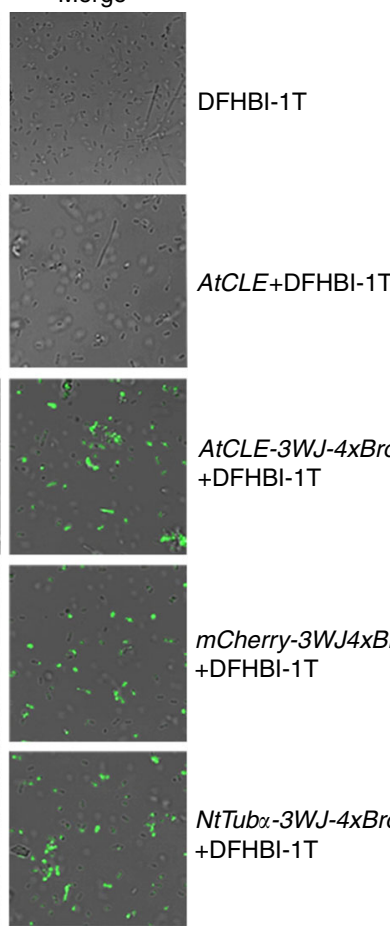
+DFHBI-1T +DFHBI-1T + DFHBI-1T b

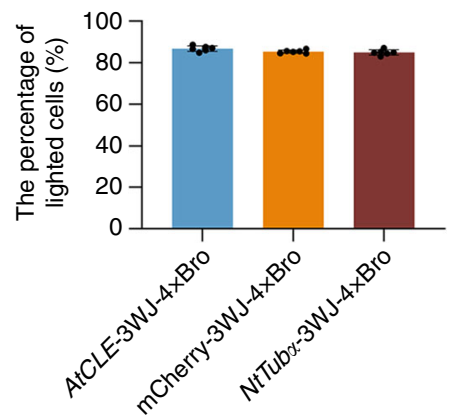

AtCLE-3WJ-4XBro

mCherry-3WJ4xBro

NtTub $\alpha-3 W J-4 x B r o$

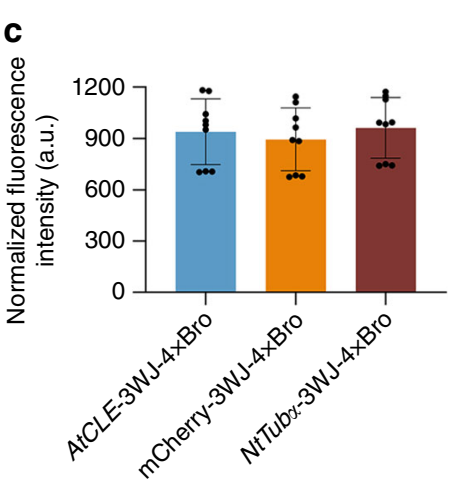

e

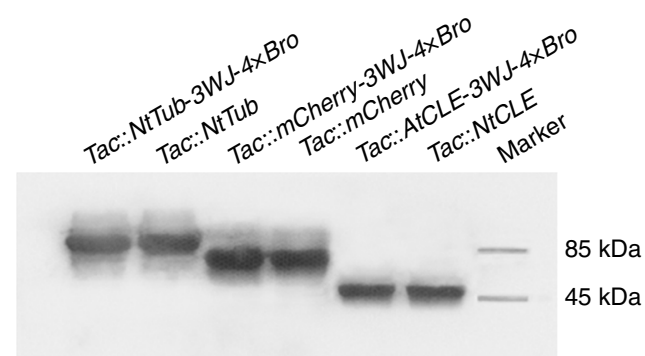

Fig. 4 Performance of $\mathbf{3 W J}-\mathbf{4} \times$ Bro in E. coli cells for reporting the expression of various genes. a Representative images of $E$. coli cells expressing three mRNAs tagged with $3 \mathrm{WJ}-4 \times$ Bro after incubation with $10 \mu \mathrm{M}$ DFHBI-1T. Scale bars, $25 \mu \mathrm{m}$. b The percentage of detectable fluorescing cells to the 500 total cells expressing each 3 WJ- $4 \times$ Bro-tagged mRNAs. Error bars, mean \pm s.d. $(n=6)$. c Fluorescence quantification of mRNAs tagged with 3 WJ- $4 \times$ Bro in cells. Error bars, mean \pm s.d. $(n=9)$. d Assessment of the integrity of three mRNAs with the 3 WJ- $4 \times$ Bro tag in E. coli cells. Total cellular RNA was extracted and separated by urea denaturing gel electrophoresis. After washing, the gel image was stained with DFHBI-1T and photographed. Red lines indicate the locations of marker bands on the gel. e Immunoblot analysis of target proteins translated from 3WJ-4 $\times$ Bro-tagged mRNAs. Source data are provided as a Source Data Underlying e. All experiments were repeated three independent times with the same conclusion. Source Data are provided as a Source Data file.

was readily observed in the nucleus but not the cytosol at $24-\mathrm{h}$ post infiltration, and bright green fluorescence gradually accumulated in the cytosol from 48- to 72 -h post infiltration, reflecting a significant lag due to the time required for the AtCLE mRNA to be translocated from the nucleus to the cytoplasm (Fig. 5f). This dynamic was consistent with the known processes of mRNA synthesis and nucleus-to-cytosol transfer, showing that the $3 \mathrm{WJ}-4 \times$ Bro/DFHBI-1T system can also be used for dynamic tracking of mRNAs.

Detection of virus-mediated RNA delivery in plant using $3 \mathrm{WJ}-$ $4 \times$ Bro. The virus-mediated RNA delivery system, which bypasses the requirement for producing transgenic plants, is widely used for gene overexpression and genome editing. TRV has been the most useful vector for this approach. However, the detection of RNA delivery still depends on RT-PCR, which is time consuming and inefficient. Consequently, we proposed a more efficient method for detecting RNA delivery using the $3 \mathrm{WJ}$ -
$4 \times$ Bro/DFHBI-1T system. Starting from a recombinant vector derived from the RNA2 genome of TRV, we created a vector to express mCherry- 3 WJ- $4 \times$ Bro mRNA under the control of the pea early browning virus (PEBV) promoter (Fig. 6a). Subsequently, the Agrobacterium cells respectively harboring the RNA1 genome and RNA2 vector were mixed and infiltrated into $N$. benthamiana leaves to reconstitute the TRV virus. We then detected fluorescence signals in both infiltrated and systemic leaves (non-infiltrated leaves of the same plant) under ultraviolet light by confocal microscopy at 5 days after infiltration.

After permeation of DFHBI-1T, infiltrated leaves showed visible green fluorescence upon exposure to ultraviolet light, but interestingly, weak fluorescence was also observed in systemic leaves (Fig. 6b), indicating that the $m$ Cherry-3WJ- $4 \times$ Bro-tagged mRNAs were delivered from the infiltrated leaves to systemic leaves by TRV. The fluorescence microscopy images further showed that $m$ Cherry-3WJ-4 $\times$ Bro-tagged mRNAs accumulated in the cytoplasm and nucleus of leaves of both types (Fig. 6b). The 


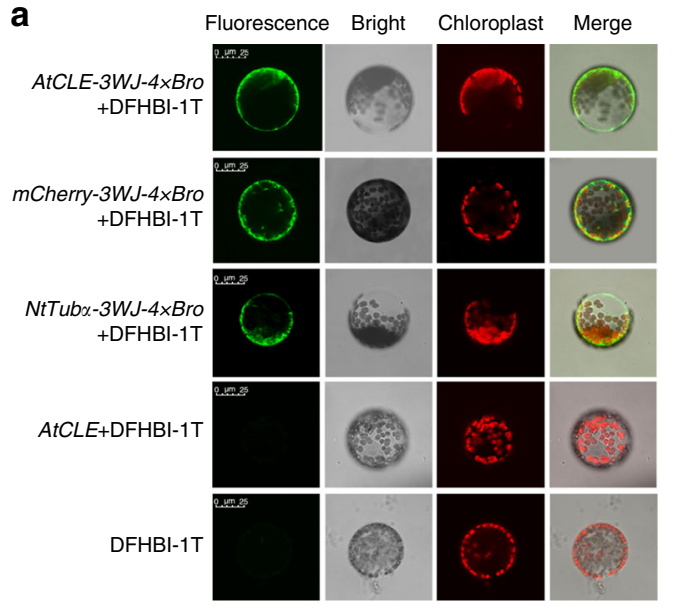

b

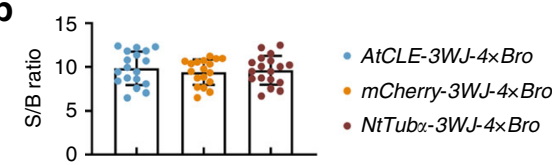

e
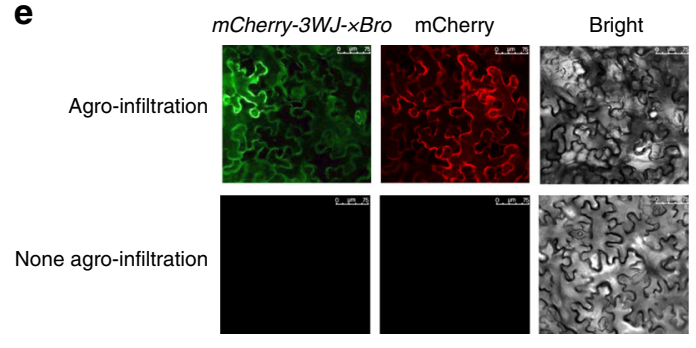

C

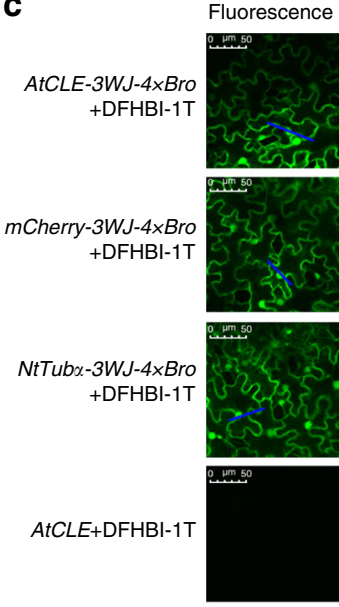

DFHBI-1T
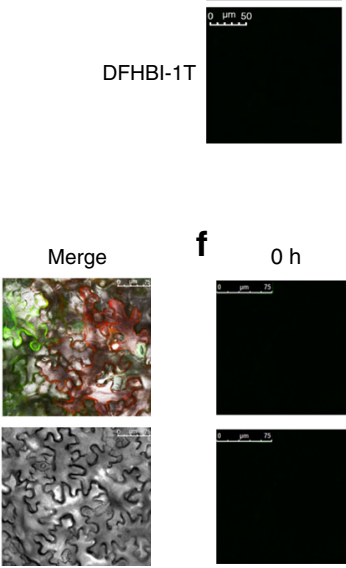
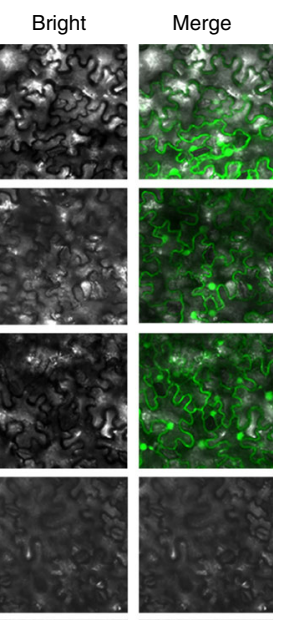

d
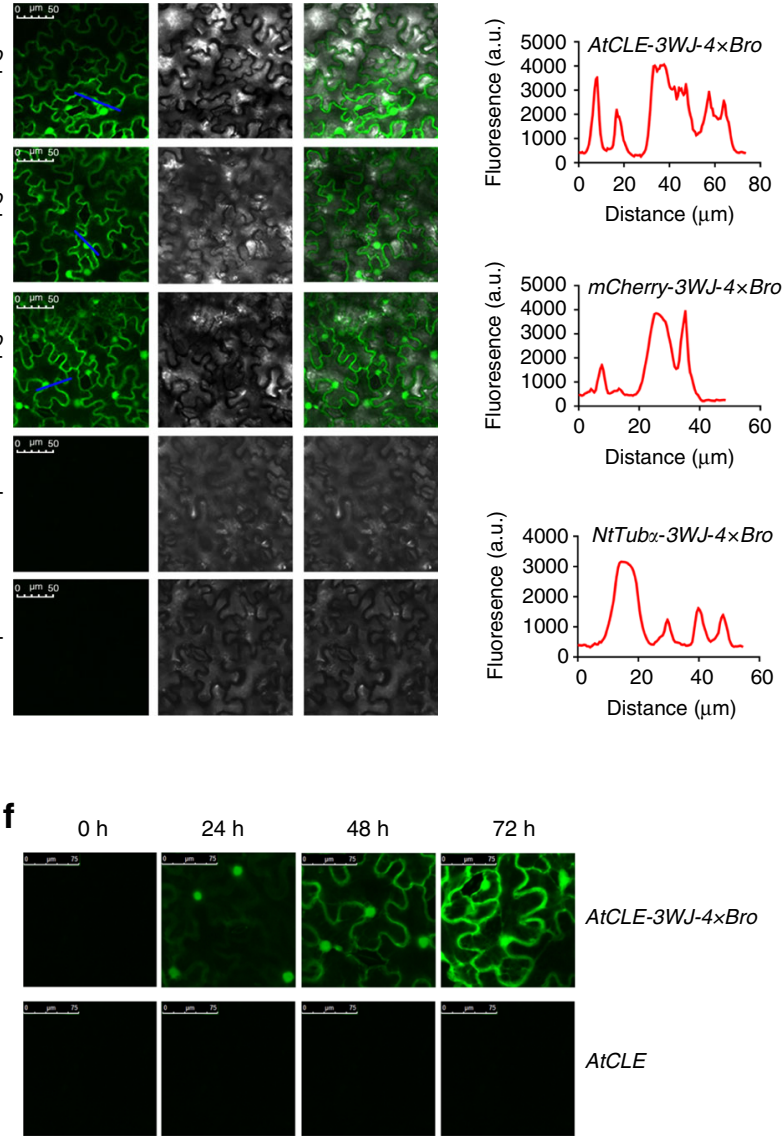

$72 \mathrm{~h}$

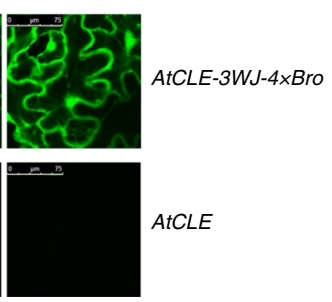

Fig. 5 Use of $\mathbf{3 W J - 4} \times$ Bro to report target genes expression in plant. a Confocal imaging of $\mathrm{N}$. benthamiana protoplasts expressing three $3 \mathrm{WJ}-4 \times$ Brotagged mRNAs. Scale bars, $25 \mu \mathrm{m}$. b Signal-to-background ratio of fluorescence in protoplasts incubated with $10 \mu \mathrm{M}$ DFHBI-1T. Error bars, mean \pm s.d. $(n=18)$. c Representative confocal images of $N$. benthamiana leaves expressing three 3 WJ- $4 \times$ Bro-tagged mRNAs. Scale bars, $25 \mu$ m. d Detection of

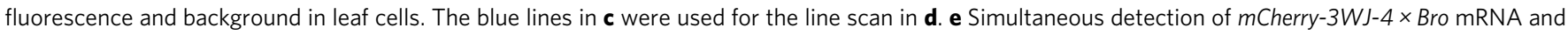
mCherry protein in N. benthamiana leaves by confocal laser microscopy. RNA of mCherry-3WJ-4 $\times$ Bro was imaged at $488-n m$ excitation and $527-n m$ emission and mCherry protein at 560-nm excitation and 610-nm emission. Scale bars, $75 \mu \mathrm{m}$. f Confocal microscopy detection of dynamic nuclear export process of NtTub $\alpha$ mRNA with 3 WJ- $4 \times$ Bro in $N$. benthamiana leaf cells at $0,24,48$, and $72-h$ post infiltration, respectively. Scale bars, $75 \mu$ m. All experiments were repeated three independent times with the same conclusion. Source Data are provided as a Source Data file.

red signal in the mCherry channel showed that mCherry protein was translated from mCherry-3WJ-4 $\times$ Bro and was mainly distributed in cytoplasm of both types of leaves (Fig. 6b). To further validate mRNA delivery, we performed RT-PCR amplification of $m$ Cherry-3WJ- $4 \times$ Bro from both inoculated and systemic leaves. A single expected band was detected in each leaf sample (Fig. 6c), and the sequencing data identified the same sequence of $m$ Cherry-3WJ-4 $\times$ Bro in two types of leaves.

Performance of $3 \mathrm{WJ}-4 \times$ Bro in plant stable transformation system. Based on the outstanding performance of the $3 \mathrm{WJ}-4 \times$ Bro/DFHBI-1T reporter system in plant transient transformation, we planned to use this reporter system for transgene identification and expression analysis of target genes in stable transformation. We selected NtTuba as a target gene and marked it with $3 \mathrm{WJ}-4 \times$ Bro at its $3^{\prime}-\mathrm{UTR}$. We then stably transformed the fusion gene NtTuba-3WJ-4 $\times$ Bro into A. thaliana. We screened 6000 transgenic $\mathrm{T}_{1}$ seeds, which were germinated on MS culture medium containing $50 \mu \mathrm{g} / \mathrm{mL}$ kanamycin, resulting in 50 kanamycin-resistant $T_{1}$ transgenic seedlings. Leaves from kanamycin-resistant seedlings were excised for positive identification by genome PCR (gPCR) and fluorescence imaging after infiltration with $30-\mu \mathrm{M}$ DFHBI-1T. gPCR analysis indicated that
45 of the 50 kanamycin-resisted seedlings were positive transgenic plants, and fluorescence imaging showed that 44 of these 50 seedlings had a bright green fluorescence (Fig. 7a, b). Moreover, all 44 fluorescent seedlings were gPCR-confirmed positive seedlings, but one of 45 gPCR-confirmed seedlings (designated $\mathrm{N}-\mathrm{F} 1$ ) showed no fluorescence under the confocal microscope (Fig. 7b). To clarify this result, we assessed NtTuba-3WJ-4 $\times$ Bro expression in N-F1 seedlings by RT-PCR. No NtTuba-3WJ-4× Bro expression was detected (Supplementary Fig. 15), indicating that NtTuba-3WJ-4 $\times$ Bro was successfully integrated into the genome but with its expression silenced. Thus, the $3 \mathrm{WJ}-4 \times \mathrm{Bro} /$ DFHBI-1T system can simultaneously and accurately report both the presence and expression of the target gene in transgenic plants.

To assess the genetic stability of $3 \mathrm{WJ}-4 \times$ Bro in transgenic offspring, we grew six $\mathrm{T}_{2}$ transgenic families derived from six $\mathrm{T}_{1}$ plants in soil and subjected $766 \mathrm{~T}_{2}$ seedlings to fluorescent identification. The results showed that $572 \mathrm{~T}_{2}$ seedlings were fluorescent and $194 \mathrm{~T}_{2}$ seedlings showed no fluorescence (Table 2). A $\chi^{2}$ test showed that the ratio of fluorescent plants to nonfluorescent plants fit the model of 3:1 Mendelian segregation in every $\mathrm{T}_{2}$ family, indicating that all six $\mathrm{T}_{1}$ transgenic plants harbor a single inserted copy of $N t T u b \alpha-3 W J-4 \times B r o$ 
a

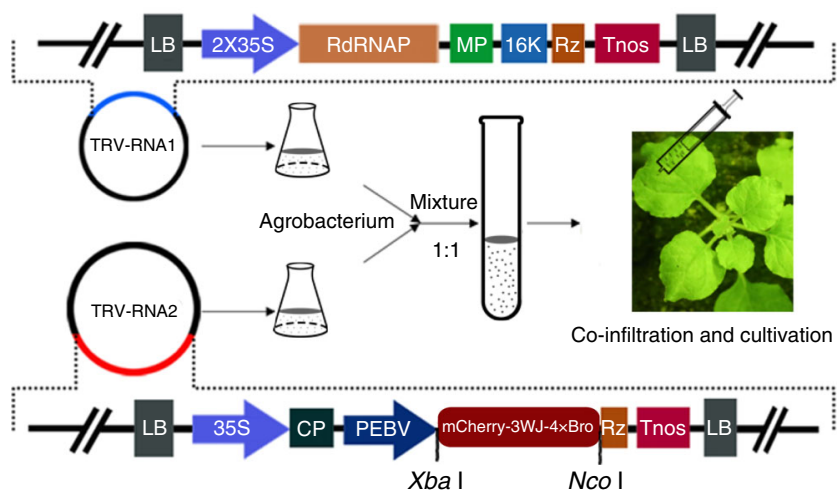

b

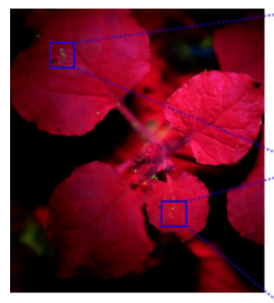

Ultraviolet exposure
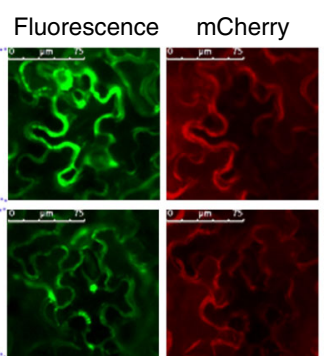

Bright

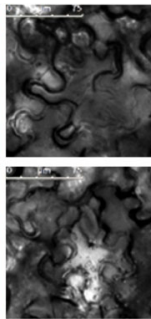

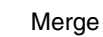

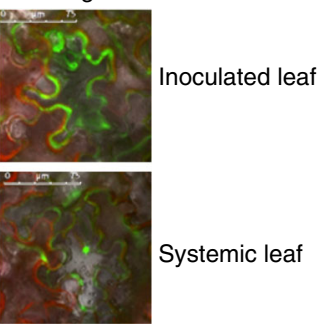

C

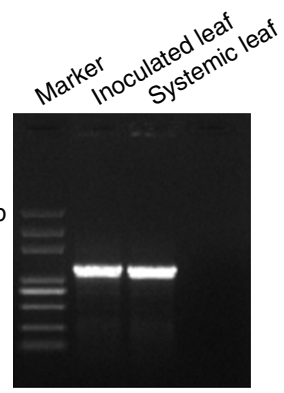

Fig. 6 Detection of long-distance RNA delivery by $\mathbf{3 W J}-\mathbf{4} \times$ Bro in $\mathbf{N}$. benthamiana. a Schematic diagram of the Agrobacterium binary vector system containing the TRV RNA1 and RNA2 genomes with some modification, as well as the experimental scheme of the TRV-mediated target RNA delivery. RNA1 contains LB (left border), $2 \times 35 \mathrm{~S}$ ( $2 \times$ CaMV 35 S promoter), RdRNAP (encoding RNA-dependent RNA polymerase), MP (encoding movement protein), 16k (encoding a cysteine-rich protein), Rz (self-cleaving ribozyme), Tnos (nopaline synthase terminator), and RB (right border). RNA2 contains LB, p35S, CP (encoding coat protein), PEBV (pea early browning virus promoter), Rz, Tnos, and RB. $m$ Cherry-3WJ-4 $\times$ Bro was cloned under the control of PEBV. The flask, tube, and syringe are drawn by software Photoshop CS6. b Fluorescence signals of $N$. benthamiana leaves under ultraviolet and confocal microscopes. Agrobacterium cultures carrying the engineered TRV RNA2 and RNA2 vectors were co-infiltrated into $\mathrm{N}$. benthamiana leaves to express and transmit 3WJ$4 \times$ Bro-tagged RNA ( $m$ Cherry-3WJ-4 $\times$ Bro). At 5 days after Agrobacterium infection, both infiltrated and systemic leaves were sampled and then incubated with 10- $\mu \mathrm{M}$ DFHBI-1T at room temperature for $20 \mathrm{~min}$. Scale bars, $75 \mu \mathrm{m}$. Three independent trials showed the same results. c Confirmation by RT-PCR of the presence of the fusion RNA ( $m$ Cherry-3WJ-4 $\times$ Bro) in both infiltrated and systemic leaves. Source Data underlying $\mathbf{b}$ are provided as a Source Data file.

(Table 2). Furthermore, RT-PCR analysis of all the $\mathrm{T}_{2}$ plants identified the 572 fluorescent plants as positive and the 194 nonfluorescent plants as negative (Table 2). These results indicated that $3 \mathrm{WJ}-4 \times$ Bro linked with the target gene was stably heritable in the transgenic progeny, making $3 \mathrm{WJ}-4 \times$ Bro an applicable gene marker for plant genetic engineering.

In addition, to investigate whether the $3 \mathrm{WJ}-4 \times$ Bro/DFHBI- $1 \mathrm{~T}$ system could report target gene expression in different tissues of transgenic plants, we imaged the leaves, root elongation zone and root tip of transgenic $A$. thaliana seedlings by fluorescence microscopy. We detected green fluorescence signals in all three tissue types, which indicate that $3 \mathrm{WJ}-4 \times$ Bro could report target gene expression in different plant tissues (Fig. $7 \mathrm{c}$ ) and thus could potentially be used to conduct tissue-specific expression analysis of target genes. Furthermore, to compare the $3 \mathrm{WJ}-4 \times \mathrm{Bro} /$ DFHBI-1T reporter system and the GFP reporter system, we detected the fluorescence intensity of $\mathrm{T}_{1}$ transgenic A. thaliana expressing NtTuba-3WJ-4 $\times$ Bro and NtTuba-GFP. Similar fluorescence intensities were observed between the two systems (Fig. 7d, e), suggesting that they possess similar sensitivity. These characteristics make $3 \mathrm{WJ}-4 \times$ Bro/DFHBI-1T a compelling reporter system for transgene identification and gene expression analysis in plants.

\section{Discussion}

Reporter systems are widely used in genetic engineering and promoter exploitation. The functions of conventional reporter systems depend on the expression of reporter genes such as FP, GUS, and $L u c$, which cause accumulation of foreign proteins in cells and hence may harm their growth and metabolism. Moreover, as has been reported before, these reporter systems have assorted limitations ${ }^{4-6}$. Consequently, we aimed to develop a protein-independent reporter system at the transcriptional level based on RNA fluorescence imaging that could be simultaneously used for identification of transgenic plants and expression analysis of target genes. To achieve this goal, it was critical to establish a precise technique for live-cell imaging of mRNA.

In our study, we set out to create fluorescence aptamers to cater to the need for RNA imaging in live plant cells and, further, to develop a reporter system for plant genetic engineering that acted at the transcriptional level. We chose to modify the F30 motif ${ }^{29}$ rather than the tRNA motif to create a scaffold based on the consideration that the tRNA scaffold is susceptible to tRNA processing enzymes when an aptamer is inserted into its anticodon loop ${ }^{30}$. In addition, we optimized the Broccoli (Bro) sequence and selected as its fluorophore DFHBI-1T, a derivative of DFHBI, that has brighter fluorescence, non-toxicity, and higher permeability 27,31 . We designed a series of $3 \mathrm{WJ}$ scaffold aptamers for mRNA imaging in vitro and in vivo. Although $3 \mathrm{WJ}-$ Bro and F30-Bro have similar MFE secondary structures (Supplementary Fig. 1), 3WJ-Bro showed higher fluorescence intensity than F30Bro (Fig. 1a, b), which we attributed to the fact that the modified $3 \mathrm{WJ}$ promoted Broccoli folding more effectively than F30 owing to differences in tertiary structure. $3 \mathrm{WJ}-4 \times$ Bro showing brightest fluorescence intensity is mainly due to the increased number of 
a

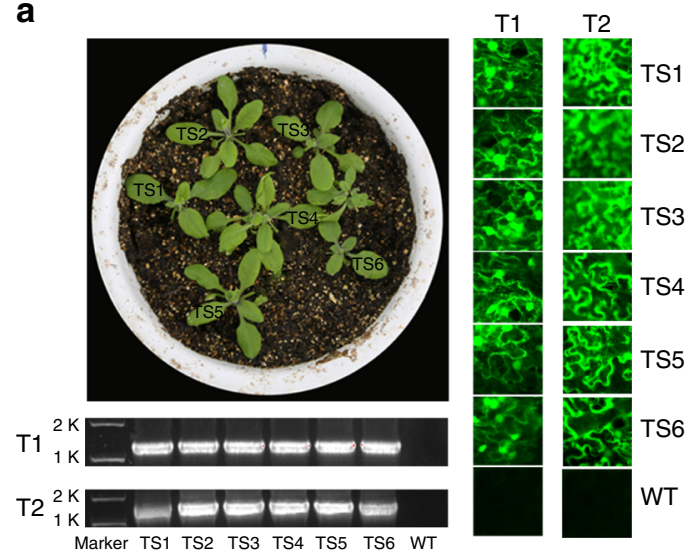

b

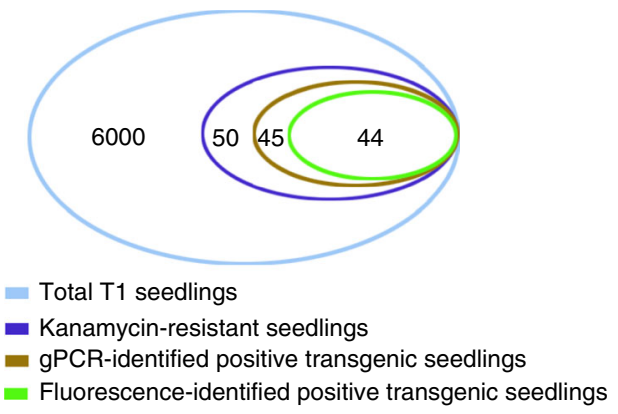

d

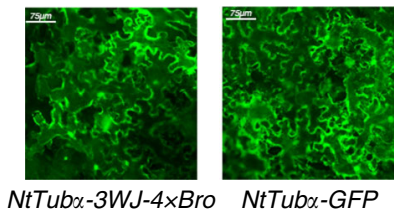

e

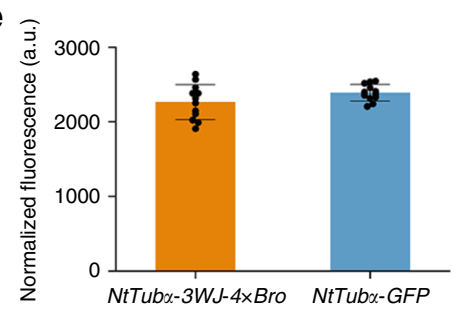

Fig. 7 Identification of transgenic A. thaliana and expression analysis of target genes using the $\mathbf{3 W J}-\mathbf{4} \times \mathbf{B r o} / \mathrm{DFBH}-1 \mathrm{~T}$ reporter system. a, $\mathbf{b}$ Identification of $T_{1}$ and $T_{2}$ transgenic $A$. thaliana. a Representative image of transgene identification by $3 \mathrm{WJ}-4 \times$ Bro fluorescence (right) and RT-PCR (bottom). TS transgenic lines, WT wild type, $T_{1}, T_{2}$ transgenic generations 1 and 2. $\mathbf{b}$ Numerical comparison of positive transgenic plants identified by the two methods above. c Expression of NtTub $\alpha-3 W J-4 \times$ Bro in different tissues of transgenic A. thaliana. The wild type was used as control. $\mathbf{d}$ The imaging comparison of the $3 \mathrm{WJ}-4 \times \mathrm{Bro} / \mathrm{DFBH}-1 \mathrm{~T}$ system (left) and the GFP reporter system (right). Scale bars, $75 \mu \mathrm{m}$. e Quantitative comparison of fluorescence intensity between the $3 \mathrm{WJ}-4 \times \mathrm{Bro} / \mathrm{DFBH}-1 \mathrm{~T}$ and GFP reporter systems. Error bars depict the mean \pm s.d. $(n=12)$. All experiments were repeated three independent times with the same conclusion. Source Data underlying a-c, e are provided as a Source Data file.

Table 2 The identification of transgenic Arabidopsis thaliana $T_{2}$ plants by fluorescence and RT-PCR.

\begin{tabular}{|c|c|c|c|c|c|c|}
\hline$T_{2}$ transgenic family & Total plant number & F-P plant number & F-N plant number & N-P plant number & $\mathbf{N}-\mathbf{N}$ plant number & $\begin{array}{l}\chi^{2} \text { value } \\
\text { for } 3: 1\end{array}$ \\
\hline$\# 1$ & 122 & 93 & 0 & 0 & 29 & 0.098 \\
\hline \#2 & 114 & 86 & 0 & 0 & 28 & 0.012 \\
\hline \#3 & 133 & 100 & 0 & 0 & 33 & 0.003 \\
\hline \#4 & 139 & 105 & 0 & 0 & 34 & 0.022 \\
\hline \#5 & 116 & 86 & 0 & 0 & 30 & 0.046 \\
\hline \# 6 & 142 & 102 & 0 & 0 & 40 & 0.761 \\
\hline Total & 766 & 572 & 0 & 0 & 194 & 0.044 \\
\hline
\end{tabular}

F-P indicates both fluorescent and RT-PCR positive plants; N-N indicates neither fluorescent nor RT-PCR positive plants; F-N indicates fluorescent but RT-PCR negative plants; N-P indicates nonfluorescent but RT-PCR positive plants.

The one-sided $\chi^{2}$ test for 3:1 of F-P plants to N-N plants were conducted with significance level at $0.01 \cdot \chi^{2}{ }_{1,0.01}=6.63$.

Broccoli units (four copies), while $3 \mathrm{WJ}-6 \times$ Bro showed lower fluorescence compared to $3 \mathrm{WJ}-4 \times \mathrm{Bro}$, which is possibly explained by that the $3 \mathrm{WJ}$ structure and linker 2 are not stable enough to effectively fold six copies of Broccoli. In addition, $3 \mathrm{WJ}-$ $4 \times$ Bro showing highest fluorescence stability is possibly attributed to its spatially stable structure enhanced by utilizing linker 1 . To investigate the aptamers' ability to image mRNA in vivo, we fused each aptamer to the $3^{\prime}$-UTR rather than $5^{\prime}$-UTR of target genes to avoid the possibility that the presence of the aptamer tertiary structure fused to the $5^{\prime}$-UTR could abolish target gene translation by hindering ribosome complex assembly ${ }^{32,33}$. Upon fusion with the mRNA AtCLE, we observed a significant decrease in fluorescence for F30-Bro, $3 \mathrm{WJ}-\mathrm{Bro}$, and $3 \mathrm{WJ}-6 \times$ Bro, but not $3 \mathrm{WJ}-4 \times$ Bro and $3 \mathrm{WJ}-2 \times$ Bro, indicating that only $3 \mathrm{WJ}-4 \times$ Bro and $3 \mathrm{WJ}-2 \times$ Bro were stable enough to tag mRNA in vitro. In addition, in vivo assays revealed that although $3 \mathrm{WJ}-2 \times$ Bro, $3 \mathrm{WJ}-$ $4 \times$ Bro, and $3 \mathrm{WJ}-6 \times$ Bro all showed good potential for mRNA imaging in E. coli, only $3 \mathrm{WJ}-4 \times$ Bro showed detectable fluorescence in $N$. benthamiana, indicating that stability of a given aptamer differed in different types of cells. This is probably the 
main reason that, although aptamers such as Mango and Corn have been used for RNA imaging in E. coli and mammalian cells, there have been no reports of their application in plant cells.

To use $3 \mathrm{WJ}-4 \times$ Bro as a marker for reporting gene expression, we investigated effects of different mRNAs on $3 \mathrm{WJ}-4 \times$ Bro in vitro and in vivo. We established that $3 \mathrm{WJ}-4 \times$ Bro could be used to image different mRNAs in both $E$. coli and plant cells, and the target proteins were detected in $E$. coli by immunoblotting and in plant cells by mCherry fluorescence (Figs. 4 and 5). Furthermore, no cleavage occurred between the mRNA and the tag in the $3 \mathrm{WJ}-4 \times$ Bro-tagged mRNA series in either type of cell, indicating that $3 \mathrm{WJ}-4 \times$ Bro fluorescence accurately reflects the expression and distribution of mRNA in live cells. By contrast, F29- and F30-scaffold Broccoli are reported to undergo differential cleavage in E. coli and mammalian cells ${ }^{25}$, which implies that the structure of the $3 \mathrm{WJ}$ scaffold plays a pivotal role in maintaining the stability of both the Broccoli aptamer and RNAs of interest. Thus, $3 \mathrm{WJ}-4 \times$ Bro is a favorable marker for reporting gene expression in plant cells without perturbation of the transcription or translation of target genes. Furthermore, our transient expression assays showed that the nuclear localization fluorescence signal of $3 \mathrm{WJ}-4 \times$ Bro-tagged mRNAs was easily detected in $N$. benthamiana leaves, but not in $N$. benthamiana protoplasts. This difference was probably due to insufficient DFHBI-1T infiltration into protoplast nuclei during the relatively short incubation time required to avoid protoplast destruction. To assess the detection sensitivity of the $3 \mathrm{WJ}-4 \times$ Bro/DFHBI- $1 \mathrm{~T}$ reporter system, we imaged the NtTub $\alpha$ mRNA tagged with $3 \mathrm{WJ}-$ $4 \times$ Bro and NtTub $\alpha$ protein with GFP under the same microscope and observed similar fluorescence intensities for both systems.

To assess the application of the $3 \mathrm{WJ}-4 \times$ Bro/DFHBI- $1 \mathrm{~T}$ system, we used it to track an RNA virus invasion (Fig. 6), a process that poses severe hazards to the growth and development of plants, including crop plants. Although numerous molecular mechanisms of virus invasion have been described ${ }^{34,35}$, direct imaging of RNA invasion dynamics is still lacking. In our study, we successfully detected the transfer of RNAs expressed by TRV from an inoculated leaf to other leaves, validating the utility of $3 \mathrm{WJ}-4 \times$ Bro as a direct imaging tool for investigating RNA virus invasion and simultaneously facilitating research into the regulatory mechanism of long-distance transport of RNA. Homoplastically, the $3 \mathrm{WJ}-4 \times$ Bro/DFHBI-1T system could also be employed to identify and monitor protein-RNA interactions ${ }^{15}$ in real time in vivo. Moreover, the system is well suited for identifying transgenic plants (Fig. 7), for which it could be a competitive alternative to traditional diagnostic PCR. During the traditional transgenic generation screening process, gPCR identification is indispensable after antibiotic selection to ensure the insertion of the complete T-DNA into the plant chromosome as opposed to vector backbone integration, which may result from target gene escape ${ }^{14}$. In addition, qPCR analysis is required to evaluate silencing efficiency and/or transcript level in the transgenic plants. However, these molecular genetic tests are costly and time consuming ${ }^{3}$. Although GFP can be used as a marker for the rapid visual screening of transgenic plants ${ }^{36,37}$, this approach requires constructing a fusion protein, which may negatively affect the biological function of target proteins. By contrast, $3 \mathrm{WJ}-$ $4 \times$ Bro provides a convenient means both to identify transgenic plants at the transcriptional level, without affecting target gene expression, and to estimate expression levels on the basis of fluorescence intensity as an alternative to qPCR. It could also be used to monitor the spatiotemporal-specific expression of a target gene during the course of a plant's lifetime.

The application of this system mainly depends on fluorescence intensity and stability. In our study, the obvious fluorescence was observed in plant cells, which is partly explained by the high accumulation transcripts of $3 \mathrm{WJ}-4 \times$ Bro-tagged gene constitutively expressed under CaMV 35S. However, endogenous gene showed a physiologically normal expression under the control of native promoter. Consequently, different types of endogenous promoter will be used to confirm potential utility of our system as a transcriptional reporter in plants in our further work. In addition, the fluorescence intensity is partly affected by the infiltration efficiency of DFHBI-1T into plant cell, which may be a limitation when comparing across different tissues or plants. This limitation could be got around if (i) plants cell membrane permeability could be changed by different solvents of DFHBI-1T solution; (ii) a derivative of DFHBI-1T, with high fluorescence and permeability, is developed.

In conclusion, we report protein-independent reporter system using a designed aptamer, $3 \mathrm{WJ}-4 \times$ Bro, and its fluorophores, DFHBI-1T, for transgene identification and gene expression analysis in plants. The $3 \mathrm{WJ}-4 \times$ Bro aptamer showed high folding efficiency and stability in plant cells, integrated into the plant genome with its target genes and co-expressed with them, and was stably inherited in future generations of transgenic lines through co-segregation with its target gene during the meiosis. Together, these properties make $3 \mathrm{WJ}-4 \times$ Bro a desirable marker for plant genetic engineering and a useful tool for various types of functional genomics studies, including explorations of protein-RNA interaction, RNA-RNA interaction, RNA virus invasion, and promoter function.

\section{Methods}

Design and clone of aptamer-related sequences. Based on the previously reported F30-Broccoli (F30-Bro), we modified the structure of the F30 scaffold to form a $3 \mathrm{WJ}$ scaffold and optimized the sequence of Broccoli (Supplementary Fig. 1). We designed a series of $3 \mathrm{WJ}-$ nBro aptamers by inserting Broccoli into two different specific sites of the $3 \mathrm{WJ}$ scaffold to construct $3 \mathrm{WJ}-$ Bro and $3 \mathrm{WJ}-2 \times$ Broccoli ( $3 \mathrm{WJ}-2 \times$ Bro) aptamers. To further improve the fluorescence intensity, we designed two specific linker sequences that provide two insert sites for $3 \mathrm{WJ}-2 \times$ Bro to construct $3 \mathrm{WJ}-4 \times$ Bro and $3 \mathrm{WJ}-6 \times$ Bro (Supplementary Fig. 1). Secondary structure predictions of these RNA aptamers were calculated using the RNAfold web server application (http://rna.tbi.univie.ac.at/cgi-bin/RNAWebSuite/RNAfold. cgi). These aptamer DNA sequences were artificially synthesized (Supplementary Table 1) and individually cloned into the plasmid pBluescript II SK between Sac II and Sac I restriction site to form the constructs of pBluescript II SK-F30-Bro and pBluescript II SK-3WJ-nBro (Supplementary Fig. 16).

Total RNAs extracted from A. thaliana and N. benthamiana leaves by E.Z.N.A. Total RNA Kit I (Omega, Cat. No. R6834) were respectively reverse transcribed to cDNA using TransScript ${ }^{\circledast}$ II One-Step gDNA Removal and cDNA Synthesis SuperMix (Transgen, Cat. No. AH311-02). The coding sequences of AtCLE (AT3G28455.1), NtTuba (LOC107826406), and mCherry were respectively amplified from $A$. thaliana cDNA, N. benthamiana cDNA, and plasmid pBI221mCherry by specific primers (AtC F/AtC R, NtT F/NtT R, and mCh F/mCh R, respectively) (Supplementary Table 2), and then cloned into the plasmid pBluescript II SK between Xba I and Sac II restriction site to form the constructs of pBluescript II SK-AtCLE/mCherry/NtTuba (Supplementary Fig. 17).

The recombinant pBluescript II SK-AtCLE-3WJ-nBro (Supplementary Fig. 18) and pBluescript II SK-AtCLE/mCherry/NtTuba-3WJ-4 × Bro (Supplementary Fig. 19) were constructed by enzyme digestion and ligation (Xba I and Sac I).

In vitro transcription and fluorescence measurement. $\mathrm{F} 30-\mathrm{Bro}, 3 \mathrm{WJ}-\mathrm{nBro}$ AtCLE-3WJ-nBro, and AtCLE/mCherry/NtTuba-3WJ-4 $\times$ Bro were amplified from their respective recombinant pBluescript II SK plasmids by PCR using the universal primers of M13 F and M13 R (Supplementary Table 2). The RNAs were transcribed in vitro using T7 RNA polymerase at $37^{\circ} \mathrm{C}$ for $16 \mathrm{~h}$ in $40 \mathrm{mM}$ Tris$\mathrm{HCl}, 30 \mathrm{mM} \mathrm{MgCl}$, $2 \mathrm{mM}$ spermidine, $1 \mathrm{mM}$ dithiothreitol (DTT), $5 \mathrm{mM}$ rNTPs, $1 \mathrm{U} / \mu \mathrm{L}$ inorganic pyrophosphatase and $4 \mathrm{U} / \mu \mathrm{L}$ T7 RNA polymerase $(\mathrm{pH}$ 7.9). The transcripts were purified on a denaturing $6 \%$ polyacrylamide gel (acrylamide: bisacrylamide $=29: 1$ ) containing $6 \mathrm{M}$ urea in TBE (Tris, borate, ethylenediaminetetraacetic acid (EDTA)) buffer ${ }^{38}$. The RNA was excised and eluted in RNA elution buffer (40 mM Tris- $\mathrm{HCl}, \mathrm{pH} 8.0,0.5 \mathrm{M}$ sodium acetate, $0.1 \mathrm{mM}$ EDTA) and precipitated with ethanol ${ }^{39}$. All transcripts were dissolved in DEPC-purified water, and the purity and concentration of RNAs were measured with a NanoDrop ND 2000 spectrophotometer. Subsequently, $1 \mu \mathrm{g}$ transcribed RNA was incubated with $10 \mu \mathrm{M}$ of fluorophore DFHBI-1T ( $Z$ )-4-(3,5-difluoro-4-hydroxybenzylidene)-2methyl-1-(2,2,2- trifluoroethyl)- $1 H$-imidazol-5(4H)-one) in $50 \mu \mathrm{L} 1 \times \mathrm{HEPES}$ buffer (PH 7.4) in a centrifuge tube at $25^{\circ} \mathrm{C}$ for $20 \mathrm{~min}$. DFHBI-1T was purchased 
from LUCERNA ${ }^{40}$.The excitation spectra of the complexes were scanned from 400 - to $540-\mathrm{nm}$ wavelengths at $527-\mathrm{nm}$ emission and the emission spectra of that were scanned from 480 to $650 \mathrm{~nm}$ at $460-\mathrm{nm}$ excitation using a fluorescence spectrophotometer (Hitachi F-7000). The extinction coefficient was calculated based on the method described by Song et al. ${ }^{41}$. The fluorescence of each aptamer-DFHBI-1T complex was imaged with a Blue Light Gel Imager ${ }^{19}$. The fluorescence intensity was measured at $488-\mathrm{nm}$ excitation and $527-\mathrm{nm}$ emission wavelengths.

Live-cell imaging mRNA in E. coli. Recombinant pBluescript II SK(+) plasmids containing F30-Bro, 3WJ-nBro, AtCLE-3WJ-nBro, and AtCLE/mCherry/NtTuba$3 \mathrm{WJ}-4 \times$ Bro were transformed into E. coli strain BL21 (DE3) cells and then grown overnight on Luria Broth solid medium containing $50 \mu \mathrm{g} / \mathrm{mL}$ ampicillin. Single positive clones identified by PCR were inoculated into Luria Broth liquid medium containing $50 \mu \mathrm{g} / \mathrm{mL}$ ampicillin and grown to exponential phase $37^{\circ} \mathrm{C}$ at $200 \mathrm{rpm}$ in a biochemical incubator, the cultures were then supplemented with $1 \mathrm{mM}$ isopropyl $\beta$-D-1-thiogalactopyranoside (IPTG) and subjected to shaking incubation at $16^{\circ} \mathrm{C}$ at $160 \mathrm{rpm}$ for the inductive expression of mRNAs with $3 \mathrm{WJ}-4 \times$ Bro tag. The cells were collected from $100 \mu \mathrm{L}$ cultures and washed once with $1 \mathrm{~mL}$ M9 medium ( $47.75 \mathrm{mM} \mathrm{Na}_{2} \mathrm{HPO}_{4}, 22.04 \mathrm{mM} \mathrm{KH}_{2} \mathrm{PO}_{4}, 8.56 \mathrm{mM} \mathrm{NaCl}, 18.7 \mathrm{mM} \mathrm{NH}_{4} \mathrm{Cl}, 2 \mathrm{mM}$ $\mathrm{MgSO}_{4}, 0.1 \mathrm{mM} \mathrm{CaCl}_{2}$ ). The washed cells were resuspended in $\mathrm{M} 9$ medium to an $\mathrm{OD}_{600}$ of 2 and incubated with $10 \mu \mathrm{M}$ DFHBI-1T for $30 \mathrm{~min}$ at $25^{\circ} \mathrm{C}$. Aliquots of $10 \mu \mathrm{L}$ were transferred to a 384 -well microtiter plate, and fluorescence intensity at $25^{\circ} \mathrm{C}$ was measured at $488-\mathrm{nm}$ excitation and $527-\mathrm{nm}$ emission wavelengths. Then, $5 \mu \mathrm{L}$ of the above cells were diluted to $20 \mu \mathrm{L}$ with M9 medium. Aliquots of $10 \mu \mathrm{L}$ were transferred to a glass slider and covered with a coverslip. The images of $E$. coli were taken at room temperature using a confocal microscope (objective $\times 63$, N.A. 1.46, excitation $488 \mathrm{~nm}$, emission $527 \mathrm{~nm}$ ) and analyzed with LAS AF Lit software ${ }^{42}$.

Prokaryotic expression and immunoblotting. AtCLE-3WJ-4 $\times$ Bro, $m$ Cherry$3 W J-4 \times B r o$, and NtTub $\alpha-3 W J-4 \times B r o$ were respectively amplified from recombinant plasmids pBluescript II SK-AtCLE/mCherry/NtTuba-3WJ- $4 \times$ Bro by specific primers of pM-At-3WJ F/pM-At-3WJ R, pM-mC-3WJ F/pM-mC-3WJ R, and pM-Nt-3WJ F/pM-Nt-3WJ R (Supplementary Table 2) and subcloned into prokaryotic expression pMol-c2x plasmids between $\mathrm{Sac}$ I and $\mathrm{Xba}$ I site (Supplementary Fig. 20). The recombinant pMol-c2x were transformed individually into $E$. coli strain Rosetta (DE3). Single positive clones identified by PCR were inoculated into Luria Broth liquid medium supplemented with $50 \mu \mathrm{g} / \mathrm{mL}$ ampicillin and grown overnight at $37^{\circ} \mathrm{C}$ at $200 \mathrm{rpm}$, and then transferred into fresh Luria Broth medium containing $50 \mu \mathrm{g} / \mathrm{mL}$ ampicillin at a ratio of $1: 100$ in a shaking incubator at $30^{\circ} \mathrm{C}$ at $180 \mathrm{rpm}$. IPTG was added to the culture medium at $1 \mathrm{mM}$ final concentration to induce protein expression and the cultures were further incubated for $10 \mathrm{~h}$ at $16^{\circ} \mathrm{C}$ at $160 \mathrm{rpm}$, when the $\mathrm{OD}_{600}$ reached $0.6-0.8$. The cells were collected by centrifugation at $6200 \times g$ for $2 \mathrm{~min}$ at $4^{\circ} \mathrm{C}$, resuspended in the buffer B1 (20 mM Tris-HCl, $500 \mathrm{mM} \mathrm{NaCl}, 1 \mathrm{mM}$ EDTA, pH 8.5), and subjected to ultrasonic disruption. The cell lysate was then separated by centrifugation at $9600 \times g$ for $10 \mathrm{~min}$ at $4{ }^{\circ} \mathrm{C}$. The precipitate was washed twice with buffer $\mathrm{B} 1$ and resuspended in the same volume of buffer B3 $(20 \mathrm{mM}$ Tris- $\mathrm{HCl}, 500 \mathrm{mM} \mathrm{NaCl}, 1 \mathrm{mM}$ EDTA, $40 \mathrm{mM}$ DTT, $\mathrm{pH} 8.5$ ). The protein expression level and purity were estimated using SDS-PAGE using a 10\% tricine SDS-PAGE gel and Coomassie blue staining ${ }^{43}$

For immunoblot analysis, the induced cells were collected and washed with icecold $1 \times$ phosphate-buffered saline. The cells were lysed with ice-cold lysis buffer (50 mM Tris-HCl (pH 7.5), $150 \mathrm{mM} \mathrm{NaCl}, 1 \mathrm{mM}$ EDTA, $1 \%$ NP-40, $2 \times$ protease inhibitor cocktail (Invitrogen)) for $30 \mathrm{~min}$ on ice. The lysate was selected by centrifugation at $11,600 \times g$ for $10 \mathrm{~min}$ at $4{ }^{\circ} \mathrm{C}$. Protein lysate samples were separated using 10\% SDS-PAGE and then transferred onto polyvinylidene difluoride membranes (EMd Millipore, Billerica, MA, USA). The membranes were blocked using $5 \%$ skim milk for $2 \mathrm{~h}$ at room temperature. After washing with $1 \times$ TBST buffer ( $50 \mathrm{mM}$ Tris base, $155 \mathrm{mM} \mathrm{NaCl}$, pH 7.6, 0.05\% Tween 20), the membranes were incubated with anti-MBP mouse monoclonal antibody (Cat. No. HT701-01, Transgen, China) at 1:1000 dilution overnight at $4{ }^{\circ} \mathrm{C}$. The membranes were washed with $1 \times$ TBST buffer, followed by goat anti-mouse IgG $(\mathrm{H}+\mathrm{L})$-HRP (Cat. No. HS201-01, Transgen, China) at dilution of 1:4000 incubation for $1 \mathrm{~h}$ at room temperature.

Live-cell imaging 3W J-4 $\times$ Bro-tagged mRNA in plant protoplasts. AtCLE$3 W J-4 \times B r o, m C h e r r y-3 W J-4 \times B r o$, and NtTub $\alpha-3 W J-4 \times$ Bro were amplified from their respective recombinant pBluescript II SK plasmids by specific primer pairs pB-At F/pB-At R, pB-mC F/pB-mC R, and pB-Nt F/pB-Nt R (Supplementary Table 2) and subcloned into pBI221 plasmids between Xba I and Sac I site (Supplementary Fig. 21) for protoplast transformation. The N. benthamiana protoplasts were obtained by the protocol described by Yoo et al. ${ }^{44}$. Then, $100 \mu \mathrm{L}$ protoplast solution $\left(1 \times 10^{4}\right.$ protoplasts $), 10 \mu \mathrm{L}$ plasmid DNA $(1 \mu \mathrm{g} / \mu \mathrm{L})$, and $110 \mu \mathrm{l} \mathrm{PEG}$ solution (PEG4000, 0.2 M mannitol, $100 \mathrm{mM} \mathrm{CaCl}_{2}$ ) were combined in $2-\mathrm{mL}$ round-bottomed microcentrifuge tubes and mixed gently. The transfection process was stopped by adding $440 \mu \mathrm{L}$ W5 solution ( $2 \mathrm{mM}$ MES (pH 5.7), $154 \mathrm{mM} \mathrm{NaCl}$, $125 \mathrm{mM} \mathrm{CaCl}_{2}$, and $5 \mathrm{mM} \mathrm{KCl}$ ) to transfection mixture after $15 \mathrm{~min}$ incubation at room temperature. The mixture was centrifuged at $100 \times g$ for $3 \mathrm{~min}$ at room temperature to remove the supernatant. The protoplasts were resuspended in $1 \mathrm{~mL}$ WI solution (4 mM MES ( $\mathrm{pH} 5.7), 0.5 \mathrm{M}$ mannitol, $20 \mathrm{mM} \mathrm{KCl}$ ) and incubated at room temperature for $12 \mathrm{~h}$. The protoplasts were centrifuged at $100 \times g$ for $2 \mathrm{~min}$ to remove the supernatant and $200 \mu \mathrm{L}$ WI solution was added to resuspend the protoplasts. DFHBI-1T at a final concentration of $10 \mu \mathrm{M}$ was added into the protoplast solution for additional 30-min incubation. Live cellular images of mRNAs tagged with $3 \mathrm{WJ}$-nBro were viewed with a confocal microscope (objective $\times 63$, N.A. 1.46, excitation $488 \mathrm{~nm}$, emission $527 \mathrm{~nm}$ ) and analyzed with LAS AF Lit software. The fluorescence intensity was measured with a fluorescence plate reader: $100 \mu \mathrm{L}$ of the treated protoplast solution above was transferred to a 384-well plate, and fluorescence intensity at $25^{\circ} \mathrm{C}$ was measured at 488 -nm excitation and $527-\mathrm{nm}$ emission wavelengths.

Expression of 3WJ-4 $\times$ Bro-tagged mRNA in N. benthamiana leaves. Recombinant pBI121 binary plasmids containing AtCLE-3WJ-4 $\times$ Bro, $m$ Cherry-3WJ- $4 \times$ Bro, and NtTuba-3WJ-4 $\times$ Bro were constructed (Supplementary Fig. 22) and transformed into Agrobacterium tumefaciens strain GV3101. Positive clones were picked from YEP agar plates containing $50 \mu \mathrm{M}$ kanamycin and $35 \mu \mathrm{M}$ rifampicin and identified by PCR, and were then inoculated into $15 \mathrm{~mL}$ YEP medium containing the same antibiotics for incubation at $28^{\circ} \mathrm{C}$ overnight at $180 \mathrm{rpm}$ in a shaker. The cells were pelleted by centrifugation at $2500 \times g$ for $10 \mathrm{~min}$ at room temperature when $\mathrm{OD}_{600}$ reached 0.8 and resuspended in agromix buffer $(10 \mathrm{mM}$ MES (pH 5.8), $10 \mathrm{mM} \mathrm{MgCl}_{2}, 200 \mu \mathrm{M}$ acetosyringone) to an $\mathrm{OD}_{600}$ at 1.0. The mixture was infiltrated into the leaves of $N$. benthamiana plants using a syringe without a needle and the infiltrated area of the leaves was marked ${ }^{45}$. After 3 days of cultivation at $25^{\circ} \mathrm{C}$ in a growth chamber with $16 \mathrm{~h} / 8 \mathrm{~h}$ (light/dark), the leaves were incubated with $10 \mu \mathrm{M}$ DFHBI-1T by infiltration for $16 \mathrm{~h}$. The mRNAs tagged with $3 \mathrm{WJ}-4 \times$ Bro were detected on the basis of their fluorescent signals using a confocal microscope (objective HCX PL APO CS $20.0 \times 0.70$ DRY UV, excitation $488 \mathrm{~nm}$, emission $527 \mathrm{~nm}$ ).

Detection of virus-mediated RNA delivery by the reporter system. The sequence of $m$ Cherry-3WJ- $4 \times$ Bro was amplified from recombinant plasmids pBluescript II SK- $m$ Cherry-3WJ-4 $\times$ Bro by specific primers TRV2-mC $\mathrm{F}$ and TRV2-mC R (Supplementary Table 2) and subcloned into pTRV2 plasmid between $\mathrm{Xba}$ I and Nco I site (Fig. 6a). The pTRV1 and recombinant pTRV2 were individually transformed into Agrobacterium GV3101. The positive Agrobacterium cells were pelleted from the medium by centrifugation and resuspended in infiltration buffer (10 $\mathrm{mM} \mathrm{MgCl}_{2}, 10 \mathrm{mM}$ MES, $200 \mu \mathrm{M}$ acetosyringone) to $\mathrm{OD}_{600} 2.0$. Agrobacterium cells harboring pTRV1 was mixed with that harboring recombinant TRV2 or nonrecombinant TRV2 (as control) at a 1:1 ratio, and each mixture was infiltrated into the leaves of $N$. benthamiana plants with a syringe without needles after $2 \mathrm{~h}$ of incubation at room temperature in darkness. The plants were grown in a growth room with a $16 \mathrm{~h} / 8 \mathrm{~h}$ (light/dark) photoperiod at a light intensity of 10,000 lux at $24^{\circ} \mathrm{C}$ for 5 days ${ }^{46}$. The leaves, including new leaves, were cut off and infiltrated with $10 \mu \mathrm{M}$ DFHBI-1T for $16 \mathrm{~h}$ and then the distribution and expression of mRNA were detected by fluorescence using a confocal microscope (objective HCX PL APO CS $20.0 \times 0.70$ DRY UV, excitation $488 \mathrm{~nm}$, emission $527 \mathrm{~nm}$ ). Total RNA was extracted from root, shoot, and leaf samples. The relative expression was determined by qRT-PCR.

Identification of stably transformed $\boldsymbol{A}$. thaliana by the reporter system. Agrobacterium GV3101 containing recombinant binary plasmids pBI121-NtTuba$3 W J-4 \times$ Bro were grown to $\mathrm{OD}_{600}$ of 1.6 at $28^{\circ} \mathrm{C}$ at $180 \mathrm{rpm}$ in a shaker. The cells were pelleted by centrifugation and resuspended in infiltration medium $(5 \%$ sucrose, $0.02 \%$ Silwet L-77) to a final $\mathrm{OD}_{600}$ of 1.0 and then incubated at room temperature in darkness for $2 \mathrm{~h}$. A. thaliana (Columbia ecotype) inflorescences were immersed in Agrobacterium solution for $10 \mathrm{~s}$ twice and covered with plastic bags to retain humidity ${ }^{47}$. After $16 \mathrm{~h}$ of cultivation in darkness, the plants were transferred to a light chamber (with a 16-h light/8-h dark photoperiod) for further growth. The seeds of $T_{0}$ generation were obtained screened on the solid MS medium containing $30 \mu \mathrm{g} / \mathrm{mL}$ kanamycin. Two-week-old kanamycin-resistant seedlings were respectively transferred to soil and MS medium for 10 days of growth. The tissues (leaf, root elongation zone, and root tip) excised for a gPCR identification using the primer pairs 35S/NOS (Supplementary Table 2) and a fluorescence detection using a confocal microscope (objective HCX PL APO CS $20.0 \times 0.70$ DRY UV, excitation $488 \mathrm{~nm}$, emission $527 \mathrm{~nm}$ ) after infiltration with $30 \mu \mathrm{M}$ DFHBI-1T. Besides, the total RNA was extracted form leaves of fluorescently transgenic plant and the RT-PCR were conducted by primers RT F/RT R

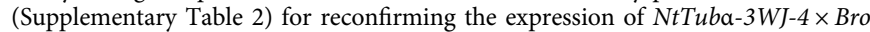
in these transgenic plants.

Integrity analysis of mRNAs with $\mathbf{3 W J} \mathbf{- 4} \times$ Bro tag. The integrity of the transcripts of $3 \mathrm{WJ}-4 \times$ Bro-tagged genes expressed in live cells was detected by in-gel imaging of fluorescence RNAs. Total RNA was extract from live cells (E. coli, A. thaliana, $N$. benthamiana) and isolated on an $8 \%$ urea gel in TBE buffer. The gel was washed three times with RNase-free water for $15 \mathrm{~min}$ to remove the urea, and stained for $20 \mathrm{~min}$ at room temperature in $10 \mu \mathrm{M}$ DFHBI-1T in $1 \times \operatorname{HEPES}(\mathrm{pH}$ 
7.4). The gel was imaged on a ChemiDoc MP (Bio-Rad) with a 488-nm excitation and $527-\mathrm{nm}$ emission ${ }^{2}$. The expected band was excised from the gel and the eluted mRNA was reverse transcribed to cDNA using a High Capacity cDNA Reverse Transcription Kit (ThermoFisher). The cDNA was then sequenced to confirm the integrity of the mRNA with $3 \mathrm{WJ}-4 \times$ Bro tag.

Reporting summary. Further information on research design is available in the Nature Research Reporting Summary linked to this article.

\section{Data availability}

Data supporting the findings of this work are available within the paper and its Supplementary Information files. A reporting summary for this Article is available as a Supplementary Information file. The datasets generated and analyzed during the current study are available from the corresponding author upon request. The data that support the findings of this study are available from the corresponding author upon reasonable request. The data underlying Figs. $1,2 \mathrm{~b}-\mathrm{e}, 3-5,6 \mathrm{~b}, 7 \mathrm{a}-\mathrm{c}$, and $7 \mathrm{e}$, as well as Supplementary Figs. 4, 5, 10, 12, and 14 are provided as a Source Data file. Source data are provided with this paper.

Received: 17 August 2019; Accepted: 2 July 2020;

Published online: 31 July 2020

\section{References}

1. Chilton, M. D. Plant genetic engineering: progress and promise. J. Agric. Food Chem. 36, 3-5 (1988).

2. Ziemienowicz, A. Plant selectable markers and reporter genes. Acta Physiol. Plant. 23, 363-374 (2001).

3. Perl, A. et al. Bacterial dihydrodipicolinate synthase and desensitized aspartate kinase: two novel selectable markers for plant transformation. Nat. Biotechnol. 11, 715-718 (1993)

4. Ow, D. W. et al. Transient and stable expression of the firefly luciferase gene in plant cells and transgenic plants. Science 234, 856-859 (1986).

5. Stewart, C. N. The utility of green fluorescent protein in transgenic plants. Plant Cell Rep. 20, 376-382 (2001).

6. Kazuki, M. et al. Functional isolation of novel nuclear proteins showing a variety of subnuclear localizations. Plant Cell 17, 389-403 (2005)

7. Quaedvlieg, N. E. et al. Fusions between green fluorescent protein and $\beta$-glucuronidase as sensitive and vital bifunctional reporters in plants. Plant Mol. Biol. 37, 715-727 (1998).

8. Lindsey, K. et al. Tagging genomic sequences that direct transgene expression by activation of a promoter trap in plants. Transgenic Res. 2, 33-47 (1993).

9. Jachoon, K. et al. A GUS/luciferase fusion reporter for plant gene trapping and for assay of promoter activity with luciferin-dependent control of the reporter protein stability. Plant Cell Physiol. 48, 1121-1131 (2007).

10. Millar, A. J., Short, S. R., Chua, N. H. \& Kay, S. A. A novel circadian phenotype based on firefly luciferase expression in transgenic plants. Plant Cell 4, 1075-1087 (1992).

11. Chinnusamy, V., Stevenson, B., Lee, B. H. \& Zhu, J. K. Screening for gene regulation mutants by bioluminescence imaging. Sci. Signal 2002, 1-10 (2002).

12. Alvarado, M. C. et al. Gene trapping with firefly luciferase in Arabidopsis. Tagging of stress-responsive genes. Plant Physiol. 134, 161-167 (2004)

13. Leeuwen, W. V. et al. The use of the luciferase reporter system forin plantagene expression studies. Plant Mol. Biol. Rep. 18, 143-144 (2000).

14. Jiang, T., Xing, B. \& Rao, J. Recent developments of biological reporter technology for detecting gene expression. Biotechnol. Genet. Eng. 25, 41-76 (2008).

15. Giani, $S$. et al. In trangenic rice, $\alpha$ - and $\beta$-tubulin regulatory sequences control GUS amount and distribution through intron mediated enhancement and intron dependent spatial expression. Transgenic Res. 18, 151-162 (2008).

16. $\mathrm{Lu}, \mathrm{J}$. et al. Gene expression enhancement mediated by the $5^{\prime}$ UTR intron of the rice rubi3 gene varied remarkably among tissues in transgenic rice plants. Mol. Genet. Genom. 279, 563-572 (2008).

17. Anthony, P. \& Susan, A. Transient expression of reporter proteins can alter plant gene expression. Plant Sci. 163, 431-437 (2002).

18. Reif, R., Haque, F. \& Guo, P. Fluorogenic RNA nanoparticles for monitoring RNA folding and degradation in real time in living cells. Nucleic Acid. Ther. 22, 428-437 (2012).

19. Paige, J. S., Wu, K. Y. \& Jaffrey, S. R. RNA mimics of green fluorescent protein. Science 333, 642-646 (2011).

20. Strack, R. L., Disney, M. D. \& Jaffrey, S. R. A superfolding Spinach2 reveals the dynamic nature of trinucleotide repeat-containing RNA. Nat. Methods 10, 1219-1224 (2013).

21. Filonov, G. S., Moon, J. D., Nina, S. \& Jaffrey, S. R. Broccoli: rapid selection of an RNA mimic of green fluorescent protein by fluorescence-based selection and directed evolution. J. Am. Chem. Soc. 136, 16299-16308 (2014).
22. Chen, $\mathrm{X}$. et al. Visualizing RNA dynamics in live cells with bright and stable fluorescent RNAs. Nat. Biotechnol. 37, 1287-1293 (2019).

23. Cawte, A. D., Unrau, P. J. \& Rueda, D. S. Live cell imaging of single RNA molecules with fluorogenic Mango II arrays. Nat. Commun. 11, 1-11 (2020)

24. Shin, I. et al. Live-cell imaging of Pol II promoter activity to monitor gene expression with RNA IMAGEtag reporters. Nucleic Acids Res 42, e90 (2014).

25. Filonov, G., Kam, C., Song, W. \& Jaffrey, S. In-Gel imaging of RNA processing using broccoli reveals optimal aptamer expression strategies. Chem. Biol. 22, 649-660 (2015).

26. Zhang, J. et al. Tandem spinach array for mRNA imaging in living bacterial cells. Sci. Rep. 5, 17295-17304 (2015).

27. Okuda, M., Fourmy, D. \& Yoshizawa, S. Use of baby spinach and broccoli for imaging of structured cellular RNAs. Nucleic Acids Res 45, 794-806 (2017).

28. Huang, K. et al. FASTmiR: an RNA-based sensor for in vitro quantification and live-cell localization of small RNAs. Nucleic Acids Res 45, e130 (2017).

29. Shu, D., Khisamutdinov, E. F., Zhang, L. \& Peixuan, G. Programmable folding of fusion RNA in vivo and in vitro driven by pRNA 3WJ motif of phi29 DNA packaging motor. Nucleic Acids Res 42, e10 (2014).

30. Ponchon, L. \& Dardel, F. Recombinant RNA technology: the tRNA scaffold. Nat. Methods 4, 571-576 (2007)

31. Svensen, N. \& Jaffrey, S. R. Fluorescent RNA aptamers as a tool to study RNAmodifying enzymes. Cell Chem. Biol. 23, 415-425 (2016).

32. Alam, K. K. et al. A fluorescent split aptamer for visualizing RNA-RNA assembly. Vivo. Acs Synth. Biol. 6, 1710-1721 (2017).

33. Trachman, R. J. et al. Structure and functional reselection of the Mango-III fluorogenic RNA aptamer. Nat. Chem. Biol. 15, 472-479 (2019).

34. Ana, M. M. H. \& Baulcombe, D. C. Tobacco rattle virus 16-kilodalton protein encodes a suppressor of RNA silencing that allows transient viral entry in meristems. J. Virol. 82, 4064-4071 (2008).

35. Dineshkumar, S. P. et al. Virus-induced gene silencing. Methods Mol. Biol. 236, 287-294 (2011).

36. Carlson, A., Letarte, J., Chen, J. \& Kasha, K. Visual screening of microsporederived transgenic barley (Hordeum vulgare L.) with green-fluorescent protein. Plant Cell Rep. 20, 331-337 (2001)

37. Yuorieva, N. O. et al. An assay for express screening of potato transformants by GFP fluorescence. Mosc. Univ. Biol. Sci. Bull. 73, 69-75 (2018).

38. Autour, A., Westhof, E. \& Ryckelynck, M. iSpinach: a fluorogenic RNA aptamer optimized for in vitro applications. Nucleic Acids Res 44, 2491-2500 (2016).

39. Warner, K. D. et al. A homodimer interface without base pairs in an RNA mimic of red fluorescent protein. Nat. Chem. Biol. 13, 1195-1201 (2017).

40. Wang, Z. et al. In-situ spatial complementation of aptamer-mediated recognition enables live-cell imaging of native RNA transcripts in real time. Angew. Chem. Int. Ed. 130, 984-988 (2018).

41. Song, W., Strack, R. L., Svensen, N. \& Jaffrey, S. R. Plug-and-play fluorophores extend the spectral properties of spinach. J. Am. Chem. Soc. 136, 1198-1201 (2014).

42. Filonov, G. S. \& Jaffrey, S. R. RNA imaging with dimeric broccoli in live bacterial and mammalian cells. Curr. Protoc. Chem. Biol. 8, 1-28 (2016).

43. Farinaz, R. \& Majid, M. B. High efficient prokaryotic expression and purification of bioactive human growth hormone using a cleavable selfaggregating tag. Gene Rep. 2018, 128-131 (2018).

44. Yoo, S. D., Cho, Y. H. \& Jen, S. Arabidopsis mesophyll protoplasts: a versatile cell system for transient gene expression analysis. Nat. Protoc. 2, 1565-1572 (2007).

45. Atul Jani, R., Nag, S. \& Setty, S. R. G. Visualization of intracellular tyrosinase activity in vitro. Bio. Protoc. 6, 1-6 (2016)

46. Ali, Z. et al. Efficient virus-mediated genome editing in plants using the CRISPR/Cas9 system. Mol. Plant 8, 1288-1291 (2015).

47. Clough, S. J. \& Bent, A. F. Floral dip: a simplified method for Agrobacteriummediated transformation of Arabidopsis thaliana. Plant J. 16, 735-743 (1998)

\section{Acknowledgements}

This work was supported by the Science and Technology Department of Sichuan Province (Grants 2017SZ0181 and 2018NZDZX0003) and the National Key R \& D Program (Grant 2018YFC1802605), People's Republic of China. This research was also supported by the Fundamental Research Funds for the Central Universities (SCU2019D013).

\section{Author contributions}

Conceptualization, Y.Z. and C.F.; funding acquisition, Y.Z. and R.W.; investigation, J.B., Y.L. and X.W.; data analysis, J.B., Y.L., X.W., S.L., M.Y., M.L. and Y.Z.; visualization, G.L., J.Y., H.Y. and M.Z.; writing original draft, J.B. and R.W.; reviewing and editing, Y.Z., C.F., W.W. and M.W

\section{Competing interests}

The authors declare no competing interests. 


\section{Additional information}

Supplementary information is available for this paper at https://doi.org/10.1038/s41467020-17497-7.

Correspondence and requests for materials should be addressed to R.W., C.F. or Y.Z.

Peer review information Nature Communications thanks Peixuan Guo, Zhanyuan Zhang and the other, anonymous, reviewer(s) for their contribution to the peer review of this work. Peer reviewer reports are available.

Reprints and permission information is available at http://www.nature.com/reprints

Publisher's note Springer Nature remains neutral with regard to jurisdictional claims in published maps and institutional affiliations. (c) (i) Open Access This article is licensed under a Creative Commons Attribution 4.0 International License, which permits use, sharing, adaptation, distribution and reproduction in any medium or format, as long as you give appropriate credit to the original author(s) and the source, provide a link to the Creative Commons license, and indicate if changes were made. The images or other third party material in this article are included in the article's Creative Commons license, unless indicated otherwise in a credit line to the material. If material is not included in the article's Creative Commons license and your intended use is not permitted by statutory regulation or exceeds the permitted use, you will need to obtain permission directly from the copyright holder. To view a copy of this license, visit http://creativecommons.org/ licenses/by/4.0/.

(C) The Author(s) 2020 